\title{
Transforming to Lorentz gauge on de Sitter
}

\author{
S. P. Miao, ${ }^{1, a)}$ N. C. Tsamis, ${ }^{2, b)}$ and R. P. Woodard ${ }^{3, c)}$ \\ ${ }^{1}$ Institute for Theoretical Physics and Spinoza Institute, Utrecht University, Leuvenlaan 4, \\ Postbus 80.195, 3508 TD Utrecht, The Netherlands \\ ${ }^{2}$ Department of Physics, University of Crete, GR-710 03 Heraklion, Greece \\ ${ }^{3}$ Department of Physics, University of Florida, Gainesville, Florida 32611, USA
}

(Received 29 July 2009; accepted 29 October 2009; published online 16 December 2009)

We demonstrate that certain gauge fixing functionals cannot be added to the action on backgrounds such as de Sitter, in which a linearization instability is present. We also construct the field-dependent gauge transformation that carries the electromagnetic vector potential from a convenient, non-de Sitter invariant gauge to the de Sitter invariant, Lorentz gauge. The transformed propagator agrees with the de Sitter invariant result previously found by solving the propagator equation in Lorentz gauge. This shows that the gauge transformation technique will eliminate unphysical breaking of de Sitter invariance introduced by a gauge condition. It is suggested that the same technique can be used to finally resolve the issue of whether or not free gravitons are de Sitter invariant. (C) 2009 American Institute of Physics. [doi:10.1063/1.3266179]

\section{INTRODUCTION}

Working out propagators is the difficult part about formulating quantum field theoretic perturbation theory on exotic backgrounds. It is typically accomplished by solving the differential equation that the propagator must obey; however, this procedure is ambiguous up to a homogeneous solution. It has long been realized that some choices for this homogeneous solution do not make the resulting Green's function into a true propagator. That is, the Green's function does not correspond to the expectation value of the time-ordered product of two free fields in the presence of any state. ${ }^{1}$

There is nothing mysterious about the problem, nor does it require field theory to understand. Consider the simple harmonic oscillator whose position as a function of time is $q(t)$ and whose Lagrangian is

$$
L=\frac{1}{2} m \dot{q}^{2}-\frac{1}{2} m \omega^{2} q^{2}
$$

The propagator equation for this system is

$$
-m\left[\left(\frac{d}{d t}\right)^{2}+\omega^{2}\right] i \Delta\left(t ; t^{\prime}\right)=i \delta\left(t-t^{\prime}\right) .
$$

The general solution to this equation, which is symmetric under interchange of $t$ and $t^{\prime}$, has three free parameters,

\footnotetext{
${ }^{\text {a)} E l e c t r o n i c ~ m a i l: ~ s . m i a o @ u u . n l . ~}$

${ }^{b}$ Electronic mail: tsamis@physics.uoc.gr.

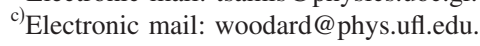




$$
i \Delta\left(t ; t^{\prime}\right)=-\frac{i}{2 m \omega} \sin \left[\omega\left|t-t^{\prime}\right|\right]+\alpha \cos (\omega t) \cos \left(\omega t^{\prime}\right)+\beta \sin \left[\omega\left(t+t^{\prime}\right)\right]+\gamma \sin (\omega t) \sin \left(\omega t^{\prime}\right) .
$$

Although any choice of $\alpha, \beta$, and $\gamma$ in (3) gives a solution to the propagator equation (2), the result is not a propagator unless they obey two inequalities,

$$
\alpha+\gamma \geq \frac{1}{2 m \omega} \text { and } \quad \alpha \gamma \geq \frac{1}{4} \beta^{2} .
$$

To see this, note first that the Heisenberg picture operator $q(t)$ can be expressed in terms of its initial position and momentum as

$$
q(t)=q_{0} \cos (\omega t)+\frac{p_{0}}{m \omega} \sin (\omega t) .
$$

For $i \Delta\left(t ; t^{\prime}\right)$ to be a propagator, there must be a state $|\psi\rangle$ such that

$$
\begin{gathered}
i \Delta\left(t ; t^{\prime}\right)=\left\langle\psi\left|T\left[q(t) q\left(t^{\prime}\right)\right]\right| \psi\right\rangle \\
=-\frac{i}{2 m \omega} \sin \left[\omega\left|t-t^{\prime}\right|\right]+\left\langle\psi\left|\frac{q_{0}^{2}}{2}\right| \psi\right\rangle \cos (\omega t) \cos \left(\omega t^{\prime}\right)+\left\langle\psi\left|\frac{q_{0} p_{0}+p_{0} q_{0}}{2 m \omega}\right| \psi\right\rangle \sin \left[\omega\left(t+t^{\prime}\right)\right] \\
+\left\langle\psi\left|\frac{p_{0}^{2}}{2 m^{2} \omega^{2}}\right| \psi\right\rangle \sin (\omega t) \sin \left(\omega t^{\prime}\right) .
\end{gathered}
$$

We can therefore identify the constants $\alpha, \beta$, and $\gamma$ as

$$
\alpha=\left\langle\psi\left|\frac{q_{0}^{2}}{2}\right| \psi\right\rangle, \quad \beta=\left\langle\psi\left|\frac{q_{0} p_{0}+p_{0} q_{0}}{2 m \omega}\right| \psi\right\rangle, \quad \gamma=\left\langle\psi\left|\frac{p_{0}^{2}}{2 m^{2} \omega^{2}}\right| \psi\right\rangle .
$$

For the ground state, one has

$$
\text { Ground state } \Rightarrow \alpha=\gamma=\frac{1}{4 m \omega} \quad \text { and } \quad \beta=0 .
$$

For a general state, the first inequality in (4) results from requiring the expectation value of the energy to be greater than or equal to $\frac{1}{2} \omega$; the second is just the Schwarz inequality.

A more subtle set of issues can arise in gauge theories. To understand them, we must digress to explain the difference between an "exact gauge" and an "average gauge." ${ }^{2}$ The former is obtained by choosing the gauge parameter to make the vector potential obey some equation at each point in space and time. This is the normal type of gauge fixing in classical field theory. Familiar examples are

$$
\begin{aligned}
& \text { Temporal gauge: } A_{0}(t, \vec{x})=0, \\
& \text { Coulomb gauge: } \vec{\nabla} \cdot \vec{A}(t, \vec{x})=0,
\end{aligned}
$$

$$
\text { Lorentz gauge: } \partial^{\mu} A_{\mu}(t, \vec{x})=-\dot{A}_{0}(t, \vec{x})+\vec{\nabla} \cdot \vec{A}(t, \vec{x})=0 .
$$

Although exact gauges can be used in quantum field theory, the more common type of gauge fixing is accomplished by adding some noninvariant term to the invariant Lagrangian. For example, the Feynman gauge Lagrangian is 


$$
\mathcal{L}=-\frac{1}{4} F_{\mu \nu} F^{\mu \nu}-\frac{1}{2}\left(\partial^{\mu} A_{\mu}\right)^{2} .
$$

The functional integral representation for this type of gauge condition can be viewed as a weighted average of exact gauges. For example, the Feynman gauge functional formalism results from imposing the exact gauge,

$$
\partial^{\mu} A_{\mu}(t, \vec{x})=f(t, \vec{x}),
$$

where $f(x)$ is a C-number field. One then functionally averages over $f(x)$ with a Gaussian weighting functional,

$$
\int[d f] \exp \left[-\frac{i}{2} \int d t \int d^{3} x f^{2}(t, \vec{x})\right] .
$$

From this discussion, it is obvious that a fairly involved set of functional changes of variables connects the exact gauge conditions of the canonical formalism to the average gauge conditions typically employed in the functional formalism. The late Sidney Coleman ${ }^{3}$ worked this out explicitly for flat space on the manifold $R^{4}$ to derive the Faddeev-Popov ansatz for this case, but the result is often assumed without justification for general metrics on any manifold. We suspect that the unjustified use of average gauge fixing is behind the dispute about the graviton propagator on de Sitter background.

It is easy to see that certain average gauges can be problematic when linearization instabilities are present. Consider flat space electrodynamics on the manifold $T^{3} \times R$. Because the spatial sections are compact, both sides of the spatially averaged, $\mu=0$ Maxwell equation must vanish separately,

$$
\partial_{\nu} F^{\nu \mu}=J^{\mu} \Rightarrow \int d^{3} x \partial_{i} F^{i 0}(t, \vec{x})=\int d^{3} x J^{0}(t, \vec{x})
$$

Because this zero charge constraint follows from the invariant field equations, it must be true as well in every exact gauge. However, naively imposing an average gauge can result in a very different theory. For example, the field equation of Feynman gauge (13) is

$$
\left[-\partial_{t}^{2}+\nabla^{2}\right] A^{\mu}(t, \vec{x})=J^{\mu}(t, \vec{x}) .
$$

This equation can be solved for any total charge. One can argue about how the problem happened, or how significant it is, but there cannot be any doubt that something went wrong.

The issues we have been discussing are relevant to a debate between cosmologists and relativists, concerning perturbative quantum gravity on de Sitter background. From the perspective of inflationary cosmology, it is natural to view de Sitter as a special case of homogeneous, isotropic, and spatially flat geometries whose invariant element in comoving coordinates takes the general form

$$
d s^{2}=-d t^{2}+a^{2}(t) d \vec{x} \cdot d \vec{x}
$$

One gets the open coordinate submanifold of de Sitter by setting the scale factor to $a(t)=e^{H t}$ with constant $H$. For any scale factor, the transverse-traceless components of the graviton field obey the same equation as the massless, minimally coupled scalar ${ }^{4}$

$$
\left[\left(\frac{\partial}{\partial t}\right)^{2}+3 H \frac{\partial}{\partial t}-\frac{\nabla^{2}}{a^{2}}\right] h_{i j}^{t t}(t, \vec{x})=0 .
$$

The power spectrum for gravitational radiation ${ }^{5}$ is proportional to the canonically normalized, superhorizon scalar mode functions $u(t, k) \sim H / k^{3 / 2}$, 


$$
\mathcal{P}_{h} \sim G \times|u(t, k)|^{2} \times k^{3} \sim G H^{2} .
$$

From scale invariance-which would be exact in de Sitter-one sees that the mode functions of physical gravitons diverge too strongly at small $k$ to give a convergent result for the Fourier mode sum of a part of the graviton propagator which must be present in any gauge. It follows that there can be no de Sitter invariant graviton propagator, just as there is no de Sitter invariant propagator for the massless, minimally coupled scalar. ${ }^{6}$

People who abhor the breaking of de Sitter invariance typically dismiss it as unphysical, but this argument cannot be accepted because the tensor power spectrum is certainly physical. (Indeed, strenuous efforts are underway to observe it!) Nor is there any support to be gained from the tiny distinction between de Sitter and primordial inflation, which typically makes the infrared behavior worse in any case. So one would think that the noninvariance of free gravitons on de Sitter must be accepted as universally as that of the massless, minimally coupled scalar. This is not so because relativists have been able to find de Sitter invariant solutions to the propagator equation in average gauges. ${ }^{7}$ Explicit solutions in what seem to be valid gauges are just as compelling as inferences from the tensor power spectrum. However, we have just seen that average gauges may not be reliable on manifolds such as de Sitter, which possess linearization instabilities.

The early recognition of a problem ${ }^{8}$ with one of these de Sitter invariant solutions led to the development of a noninvariant, average gauge. ${ }^{9,10}$ The associated propagator has been shown to obey the Ward identities at tree order ${ }^{11}$ and one loop; ${ }^{12}$ and the only fully renormalized, dimensionally regulated loop results for quantum gravity on de Sitter background have been obtained using it. ${ }^{13-15}$ Although the gauge fixing functional of this propagator is not de Sitter invariant, it does preserve the one-parameter subgroup of dilatations,

$$
\begin{gathered}
t \rightarrow t-\frac{1}{H} \ln (k), \\
\vec{x} \rightarrow k \vec{x},
\end{gathered}
$$

so the fact that the propagator breaks dilatation invariance cannot be blamed on the gauge. Moreover, Kleppe showed that the propagator's de Sitter breaking is physical by the standard technique of supplementing naive de Sitter transformations with a compensating gauge transformation to restore the noninvariant gauge condition. ${ }^{16}$

As with the cosmological power spectrum, one would think these results decisive, but the interest in a de Sitter invariant graviton propagator persists. ${ }^{17}$ What seems to be necessary to settle the issue is two things:

- a proof that the average gauges for which de Sitter invariant solutions have been found are not valid and

- an explicit construction of the graviton propagator in an exact, de Sitter invariant gauge, which is valid over the full de Sitter manifold.

Of course, the imposition of a de Sitter invariant gauge would make the propagator equation de Sitter invariant, but that does not imply a de Sitter invariant solution for the graviton propagator any more than it does for the massless, minimally coupled scalar propagator, which obeys an invariant equation but is not invariant. ${ }^{6}$ If the graviton propagator in an exact gauge breaks de Sitter invariance, then the breaking must be accepted as physical. This would not only resolve a contentious dispute, but the resulting propagator might be easier to use and it would reduce the number of counterterms. ${ }^{14,15}$

In Sec. II of this paper, we give a proof that the average de Sitter invariant gauges are not valid; in subsequent sections, we develop the machinery for constructing the propagator in exact de Donder gauge. The technique for our construction is to find the field-dependent gauge transformation $\xi_{\mu}[h]$, which enforces the gauge condition 


$$
h_{\mu \nu}^{\prime}=h_{\mu \nu}-2 \xi_{\mu ; \nu}[h] \quad \text { such that } g^{\rho \sigma}\left[h_{\mu \rho ; \sigma}^{\prime}-\frac{1}{2} h_{\rho \sigma ; \mu}^{\prime}\right]=0 .
$$

(In this and subsequent formulas, $g_{\mu \nu}$ stands for the spacelike background de Sitter metric and a semicolon denotes covariant differentiation with respect to this background.) Then, we use this transformation to carry the existing noninvariant propagator ${ }^{9,10}$ into de Donder gauge. If the de Sitter breaking of the existing propagator is completely due to the noninvariant gauge, then the de Donder gauge result should be invariant; if the de Sitter breaking is physical, then it should persist after the transformation.

As a warm-up for de Sitter gravity, here we carry out the same exercise for de Sitter electromagnetism. That is, we find the field-dependent gauge transformation $\theta[A]$, which imposes exact Lorentz gauge

$$
A_{\mu}^{\prime}=A_{\mu}-\partial_{\mu} \theta[A] \text { such that } \partial_{\mu}\left(\sqrt{-g} g^{\mu \nu} A_{\nu}^{\prime}\right)=0 .
$$

Then, we use this transformation on the photon propagator in a noninvariant average gauge. ${ }^{10,18}$ Because we already know the unique, de Sitter invariant solution for the propagator equation in Lorentz gauge, ${ }^{19}$ obtaining this known solution by transformation demonstrates that the technique will remove de Sitter breaking that arises from using a noninvariant gauge condition. The simpler setting of electromagnetism and the close relation between the noninvariant electromagnetic and gravitational gauges should also teach us much of value for the main project.

This paper consists of six sections of which the first is ending. In Sec. II we review the functional changes in variables which carry one from an exact, canonical gauge to a covariant, average gauge, with special attention to what goes wrong when linearization instabilities are present. The context is flat space electrodynamics on the $D$-dimensional manifolds $R^{D}$ and $T^{D-1}$ $\times R$. In Sec. III, we switch to $D$-dimensional de Sitter and carry out the field-dependent gauge transformation that enforces exact Lorentz gauge. Of course, this gauge transformation is ambiguous up to a homogeneous solution, which is uniquely determined by de Sitter invariance but which we leave unspecified at this stage. In Sec. IV, we describe the unique de Sitter invariant solution that was found by solving the propagator equation in Lorentz gauge. ${ }^{19}$ In Sec. V, we show how the hitherto unspecified homogeneous part of the gauge transformation from Sec. III can be chosen to make the two propagators agree. Our conclusions comprise Sec. VI.

\section{DERIVING AVERAGE GAUGES}

The purpose of this section is to explain how one derives average gauges from the exact gauges of the canonical formalism. We shall take flat space quantum electrodynamics (QED) as the object of study and first sketch the technique for passing from Coulomb gauge to Feynman gauge on the manifold $R^{D}$. We then consider the same process for the manifold $T^{D-1} \times R$ to show explicitly why full Feynman gauge cannot be imposed.

The QED Lagrangian is

$$
\mathcal{L}=-\frac{1}{4} F_{\mu \nu} F^{\mu \nu}+\bar{\psi} \gamma^{\mu}\left(i \partial_{\mu}-e A_{\mu}\right) \psi-m \bar{\psi} \psi
$$

The canonical dynamical variables of Coulomb gauge $(\vec{\nabla} \cdot \vec{A}(t, \vec{x})=0)$ are the transverse components of the vector potential, $A_{i}^{T}(t, \vec{x})$, and the electric field, $E_{i}^{T}(t, \vec{x})$, as well as $\psi(t, \vec{x})$ and $\bar{\psi}(t, \vec{x})$. The $\mu=0$ component of the vector potential is not an independent dynamical variable but a nonlocal functional $\Phi[\bar{\psi}, \psi](t, \vec{x})$ of the charged fields

$$
A_{0}=\Phi[\bar{\psi}, \psi] \equiv-\frac{1}{\nabla^{2}}\left[e \bar{\psi} \gamma^{0} \psi\right]
$$

Moreover, the Hamiltonian density of Coulomb gauge is

$$
\mathcal{H}=\frac{1}{2} E_{i}^{T} E_{i}^{T}+\frac{1}{4} F_{i j} F_{i j}-\frac{1}{2} \Phi \nabla^{2} \Phi+\bar{\psi}\left(-i \gamma^{j} \partial_{i}+e \gamma^{j} A_{i}^{T}+m\right) \psi
$$


The usual connection between the canonical and the functional formalisms is made through the vacuum expectation values of $T^{*}$-ordered products of operators. ( $T^{*}$-ordering is the same as time-ordering except that the derivatives are taken outside the ordering.) Suppose $O\left[\bar{\psi}, \psi, E^{T}, A^{T}\right]$ represents an arbitrary functional of the canonical operators. The fundamental relation between the canonical and the functional integral formalisms is

$$
\left\langle\Omega\left|T^{*}\left(\mathcal{O}\left[\bar{\psi}, \psi, E^{T}, A^{T}\right]\right)\right| \Omega\right\rangle=\int[d \bar{\psi}][d \psi]\left[d E^{T}\right]\left[d A^{T}\right] e^{i \int d^{D} x\left[i \bar{\psi} \gamma^{0} \dot{\psi}+E_{i}^{T} \dot{A}_{i}^{T}-\mathcal{H}\right]} \mathcal{O}\left[\bar{\psi}, \psi, E^{T}, A^{T}\right]
$$

Because they will play no role in our analysis, we have suppressed the initial and final state wave functionals.

We henceforth denote expression (28) as $\langle\mathcal{O}\rangle$. A seven-step process of functional manipulations carries it to Feynman gauge on $R^{D}$.

(1) Perform the Gaussian integrals over the transverse electric field,

$$
\int\left[d E^{T}\right] e^{i \int d^{D} x\left[E_{i}^{T} \dot{A}_{i}^{T}-1 / 2 E_{i}^{T} E_{i}^{T}\right]} \mathcal{O}\left[\bar{\psi}, \psi, E^{T}, A^{T}\right]=\mathcal{O}^{\prime}\left[\bar{\psi}, \psi, A^{T}\right] e^{i \int d^{D} x 1 / 2 \dot{A}_{i}^{T} \dot{A}_{i}^{T}},
$$

where $\mathcal{O}^{\prime}\left[\bar{\psi}, \psi, A^{T}\right]$ is $\mathcal{O}\left[\bar{\psi}, \psi, \dot{A}^{T}, A^{T}\right]$ plus the delta function correlator terms that arise from eliminating the various pairings of $E^{T}$, s.

(2) Restore the longitudinal component of the vector potential,

$$
\int\left[d A^{T}\right]=\int[d \vec{A}] \delta[\vec{\nabla} \cdot \vec{A}] \sqrt{\operatorname{det}\left(-\nabla^{2}\right)} .
$$

Note that this gives the square root of the Faddeev-Popov determinant for Coulomb gauge.

Restore the temporal component of the vector potential,

$$
e^{i \int d^{D} x 1 / 2 \Phi \nabla^{2} \Phi}=\sqrt{\operatorname{det}\left(-\nabla^{2}\right)} \times \int\left[d A_{0}\right] e^{i \int d^{D} x\left[1 / 2 \partial_{i} A_{0} \partial_{i} A_{o}-\bar{\psi} \gamma^{0} e A_{0} \psi\right]} .
$$

Note that this gives the remaining bit of the Faddeev-Popov determinant. At this stage, $\langle\mathcal{O}\rangle$ takes the form,

$$
\begin{aligned}
& \langle\mathcal{O}\rangle=\int[d \bar{\psi}][d \psi][d A] \delta[\vec{\nabla} \cdot \vec{A}] \operatorname{det}\left(-\nabla^{2}\right) \\
& \times e^{i \int d^{D} x\left[1 / 2 \dot{A}_{i} \dot{A}_{i}+1 / 2 \partial_{i} A_{0} \partial_{i} A_{0}-1 / 4 F_{i j} F_{i j}+\bar{\psi}\left(i \gamma^{\mu} \partial_{\mu}-\gamma^{\mu} e \mu_{\mu}-m\right) \psi\right]} \mathcal{O}^{\prime \prime}[\bar{\psi}, \psi, A],
\end{aligned}
$$

where $\mathcal{O}^{\prime \prime}[\bar{\psi}, \psi, A]$ is $\mathcal{O}^{\prime}[\bar{\psi}, \psi, \vec{A}]$ with possible factors of $\Phi[\bar{\psi}, \psi]$ replaced by $A_{0}$ (if desired, it is not necessary) and with the addition of appropriate correlator terms for pairings of $A_{0}$ 's.

(4) Express the integrand as an invariant. Any good gauge can be used to express an arbitrary functional of the fields as a gauge invariant which happens to agree with the original functional when the gauge condition is obeyed. ${ }^{2,20}$ We do this for the action and for the operator $\mathcal{O}^{\prime \prime}[\bar{\psi}, \psi, A]$,

$$
\begin{gathered}
\delta[\vec{\nabla} \cdot \vec{A}] \times e^{i \int d^{D} x\left[1 / 2 \dot{A}_{i} \dot{A}_{i}+1 / 2 \partial_{i} A_{0} \partial_{i} A_{0}\right]}=\delta[\vec{\nabla} \cdot \vec{A}] \times e^{i \int d^{D} x 1 / 2 F_{0 i} F_{0 i}}, \\
\delta[\vec{\nabla} \cdot \vec{A}] \times \mathcal{O}^{\prime \prime}[\bar{\psi}, \psi, A]=\delta[\vec{\nabla} \cdot \vec{A}] \times \mathcal{O}_{\text {inv }}[\bar{\psi}, \psi, A] .
\end{gathered}
$$

After invariantizing in this way, our expression for $\langle\mathcal{O}\rangle$ is 


$$
\langle\mathcal{O}\rangle=\int[d \bar{\psi}][d \psi][d A] \delta[\vec{\nabla} \cdot \vec{A}] \operatorname{det}\left(-\nabla^{2}\right) e^{i S_{\mathrm{inv}} \mathcal{O}_{\mathrm{inv}}}
$$

(5) Make a functional change of variables to Lorentz gauge. Consider the field-dependent gauge transformation

$$
\begin{gathered}
A_{\mu}^{\prime}=A_{\mu}-\partial_{\mu} \theta_{1}[A], \\
\psi^{\prime}=e^{i e \theta_{1}[A]} \times \psi, \\
\bar{\psi}^{\prime}=e^{-i e \theta_{1}[A]} \times \bar{\psi},
\end{gathered}
$$

where the gauge parameter is

$$
\theta_{1}[A]=-\frac{1}{\partial^{2}} \dot{A}_{0}
$$

Because $S_{\text {inv }}$ and $\mathcal{O}_{\text {inv }}$ are gauge invariant, only the gauge fixing delta functional and the measure will change. To get them, note that the inverse transformation for the vector potential is

$$
A_{\mu}=A_{\mu}^{\prime}-\partial_{\mu} \frac{1}{\nabla^{2}} \dot{A}_{0}^{\prime}
$$

It follows that the Coulomb gauge condition on $A_{\mu}$ implies the Lorentz gauge condition on $A_{\mu}^{\prime}$,

$$
\partial_{i} A_{i}=\partial^{\mu} A_{\mu}^{\prime} .
$$

Also, the functional Jacobian converts the Faddeev-Popov determinant to the one appropriate for Lorentz gauge

$$
[d A] \operatorname{det}\left(-\nabla^{2}\right)=\left[d A^{\prime}\right] \operatorname{det}\left(-\partial^{2}\right) .
$$

We can therefore write $\langle\mathcal{O}\rangle$ as

$$
\langle\mathcal{O}\rangle=\int\left[d \bar{\psi}^{\prime}\right]\left[d \psi^{\prime}\right]\left[d A^{\prime}\right] \delta\left[\partial^{\mu} A_{\mu}^{\prime}\right] \operatorname{det}\left(-\partial^{2}\right) e^{i S_{\text {inv }}} \mathcal{O}_{\text {inv }} .
$$

(6) Add an inhomogeneous, $\mathrm{C}$-number term to the gauge fixing functional. Make an additional change in variable,

$$
\begin{aligned}
A_{\mu}^{\prime \prime} & =A_{\mu}^{\prime}-\partial_{\mu} \theta_{2}[f], \\
\psi^{\prime \prime} & =e^{i e \theta_{2}[f]} \times \psi, \\
\bar{\psi}^{\prime \prime} & =e^{-i e \theta_{2}[f]} \times \bar{\psi},
\end{aligned}
$$

where the gauge parameter is defined in terms of an arbitrary C-number field $f(t, \vec{x})$,

$$
\theta_{2}[f]=\frac{1}{\partial^{2}} f
$$

After the transformation, we have

$$
\langle\mathcal{O}\rangle=\int\left[d \bar{\psi}^{\prime \prime}\right]\left[d \psi^{\prime \prime}\right]\left[d A^{\prime \prime}\right] \delta\left[\partial^{\mu} A_{\mu}^{\prime \prime}-f\right] \operatorname{det}\left(-\partial^{2}\right) e^{i S_{\mathrm{inv}} \mathcal{O}_{\mathrm{inv}}}
$$


(7) Functionally average over the inhomogeneous term. By construction, $\langle\mathcal{O}\rangle$ has no dependence on the function $f(t, \vec{x})$. It is therefore unchanged if we multiply by a normalized Gaussian and functional integrate over $f$,

$$
\begin{gathered}
\langle\mathcal{O}\rangle=\int[d f] e^{i \int d^{D} x 1 / 2 f^{2}} \times\langle\mathcal{O}\rangle \\
=\int\left[d \bar{\psi}^{\prime \prime}\right]\left[d \psi^{\prime \prime}\right]\left[d A^{\prime \prime}\right] \operatorname{det}\left(-\partial^{2}\right) e^{i S_{\text {Feynman }}} \mathcal{O}_{\text {inv }} .
\end{gathered}
$$

This is the Feynman gauge functional formalism we sought.

Let us see how the sequence of functional manipulations described above changes when the noncompact spatial manifold $R^{D-1}$ is replaced by the compact manifold $T^{D-1}$. A major difference is that Fourier integrals become discrete sums. Suppose the range of each spatial coordinate is $-L \leq x^{i}<+L$ for $i=1,2, \ldots(D-1)$. Then, any function $f(t, \vec{x})$ can be expressed as a discrete Fourier sum,

$$
f(t, \vec{x})=\sum_{\vec{n} \in Z^{D-1}} f_{\vec{n}}(t) e^{i k \vec{n} \cdot \vec{x}},
$$

where $k \equiv \pi / L$ is the fundamental wave number. Note that the action of $\nabla^{2}$ on any such function annihilates the $\vec{n}=0$ mode,

$$
\nabla^{2} f(t, \vec{x})=-\sum_{\vec{n} \in Z^{D-1}}(k n)^{2} f_{\vec{n}}(t) e^{i \pi L^{-1} \vec{n} \cdot \vec{x}} .
$$

Hence, the instantaneous Coulomb potential can only be defined for configurations of $\bar{\psi}(t, \vec{x})$ and $\psi(t, \vec{x})$, which have zero total charge (this is the linearization stability constraint!) and the resulting potential has no $\vec{n}=0$ mode,

$$
\begin{gathered}
\Phi(t, \vec{x})=-\frac{1}{\nabla^{2}}\left[\bar{\psi} \gamma^{0} e \psi\right](t, \vec{x}) \\
=\sum_{\vec{n} \neq 0} \frac{1}{(k n)^{2}(2 L)^{D-1} \int d^{D-1} x^{\prime} e^{i k \vec{n} \cdot\left(\vec{x}-\vec{x}^{\prime}\right)} \bar{\psi}\left(t, \vec{x}^{\prime}\right) \gamma^{0} e \psi\left(t, \vec{x}^{\prime}\right) .}
\end{gathered}
$$

Of course, this means that when $A_{0}(t, \vec{x})$ is restored in step (3), it cannot contain any $\vec{n}=0$ mode.

Another important change concerns restoring the longitudinal part of the vector potential in step (2). Under a gauge transformation, the Fourier mode $\vec{A}_{\vec{n}}(t)$ goes to

$$
\vec{A}_{\vec{n}}^{\prime}(t)=\vec{A}_{\vec{n}}(t)-i k n \theta_{\vec{n}}(t)
$$

It follows that all three vector components of $\vec{A}_{0}(t)$ are physical. (In the noninteracting theory they would behave like free quantum mechanical particles rather than harmonic oscillators.) A second consequence is that the Coulomb gauge delta functional lacks a $\vec{n}=0$ mode,

$$
\delta[\vec{\nabla} \cdot \vec{A}]=\prod_{t \in R} \prod_{\vec{n} \neq 0} \delta\left(k \vec{n} \cdot \vec{A}_{\vec{n}}(t)\right)
$$

To recapitulate, the changes associated with working on the compact spatial manifold $T^{D-1}$ are as follows.

- the field $A_{0}(t, \vec{x})$ contains no $\vec{n}=0$ mode and

- the Coulomb gauge delta functional contains no $\vec{n}=0$ mode. 
It is immediately obvious that while we can still make the functional change of variables in step (7) to enforce exact Lorentz gauge, neither the resulting $A_{0}^{\prime}(t, \vec{x})$ nor the Lorentz gauge delta functional will contain an $\vec{n}=0$ mode. It follows that there is no valid way to add the $\vec{n}=0$ mode of the Feynman gauge fixing term. So the result is just what we expected: adding the full Feynman gauge fixing term is incorrect, and all conclusions drawn from this formalism are suspect. It should be clear that the same sort of problem must occur as well in de Sitter and for gravitons as well as for electromagnetism, so we now have a proof that the average gauges for which de Sitter invariant solutions have been found are not valid.

Let us return to the context of flat space electromagnetism on the manifold $T^{D-1} \times R$ and consider what goes wrong if the problem we have just demonstrated is ignored and the covariant gauge fixing term (with $\vec{n}=0$ mode) is erroneously added to the invariant action. In that case, there is an extra homogeneous contribution to $A_{0}(t, \vec{x})$, which should not be present, and a corresponding extra contribution to propagator. For a point charge $q$, this extra term produces a homogeneous contribution to $A_{0}(t, \vec{x})$, which grows like $t^{2}$,

$$
A_{0}(t, \vec{x})=\frac{q t^{2}}{2^{D} L^{D-1}}+\text { higher modes. }
$$

One might object that the extra term is harmless because it makes no contribution to the electric field; however, the undifferentiated potential does contribute to the interaction energy. A special case of some interest in QED is the interaction of a particle with its own force fields. In this context, it has been noted that using the de Sitter invariant, Feynman gauge propagator ${ }^{21}$ (which must also contain a spurious homogeneous part) results in on-shell singularities for the one loop self-mass-squared of a charged scalar. ${ }^{18}$ Just as the analysis of this section suggests, these on-shell singularities disappear either

- when using a non-de Sitter invariant gauge on the open coordinate submanifold (which has no linearization instability) ${ }^{18}$ or

- when using the de Sitter invariant Lorentz gauge propagator (which is exact). ${ }^{22}$

It should be emphasized that there is no mistake in the Allen-Jacobson solution for the Feynman gauge propagator ${ }^{21}$ it is the gauge fixing functional which is at fault.

\section{IMPOSING LORENTZ GAUGE ON DE SITTER}

The purpose of this section is to make a field-dependent gauge transformation that carries the photon propagator from a non-de Sitter invariant average gauge, defined on the open coordinate submanifold, to the de Sitter invariant, exact Lorentz gauge, which can be extended to the entire de Sitter manifold. We begin by reviewing the geometry and coordinate system, and then we give the noninvariant gauge condition and the associated propagator. The next step is making the transformation. Of course, this is ambiguous up to surface terms, which we leave to be specified in Sec. V. We close by decomposing the transformed propagator (without the homogeneous contributions) into two convenient pieces.

We work on the open conformal coordinate submanifold of $D$-dimensional de Sitter space. A space-time point $x^{\mu}$ can be decomposed into its temporal $\left(x^{0}\right)$ and spatial $x^{i}$ components, which take values in the ranges

$$
-\infty<x^{0}<0 \text { and }-\infty<x^{i}<+\infty .
$$

In these coordinates, the invariant element is

$$
d s^{2} \equiv g_{\mu \nu} d x^{\mu} d x^{\nu}=a_{x}^{2} \eta_{\mu \nu} d x^{\mu} d x^{\nu}
$$

where $\eta_{\mu \nu}$ is the Lorentz metric and $a_{x}=-1 / H x^{0}$ is the scale factor. The parameter $H$ is known as the "Hubble constant." 
Most of the various propagators between points $x^{\mu}$ and $z^{\mu}$ can be expressed in terms of the de Sitter length function $y(x ; z)$,

$$
y(x ; z) \equiv\|\vec{x}-\vec{z}\|^{2}-\left(\left|x^{0}-z^{0}\right|-i \epsilon\right)^{2} .
$$

Except for the factor of $i \epsilon$ (whose purpose is to enforce Feynman boundary conditions), the function $y(x ; z)$ is closely related to the invariant length $\ell(x ; z)$ from $x^{\mu}$ to $z^{\mu}$,

$$
y(x ; z)=4 \sin ^{2}\left(\frac{1}{2} H \ell(x ; z)\right) .
$$

Because $y(x ; z)$ is a de Sitter invariant, so too are covariant derivatives of it,

$$
\begin{gathered}
\frac{\partial y(x ; z)}{\partial x^{\mu}}=H a_{x}\left(y \delta_{\mu}^{0}+2 a_{z} H \Delta x_{\mu}\right), \\
\frac{\partial y(x ; z)}{\partial z^{\nu}}=H a_{z}\left(y \delta_{\nu}^{0}-2 a_{x} H \Delta x_{\nu}\right), \\
\frac{\partial^{2} y(x ; z)}{\partial x^{\mu} \partial z^{\nu}}=H^{2} a_{x} a_{z}\left(y \delta_{\mu}^{0} \delta_{\nu}^{0}+2 a_{z} H \Delta x_{\mu} \delta_{\nu}^{0}-2 a_{x} \delta_{\mu}^{0} H \Delta x_{\nu}-2 \eta_{\mu \nu}\right) .
\end{gathered}
$$

Here and subsequently, $\Delta x_{\mu} \equiv \eta_{\mu \nu}(x-z)^{\nu}$.

Electromagnetism is conformally invariant in $D=4$ dimensions, which means that it takes the same form in conformal coordinates as in flat space. This is obvious from the gauge invariant Lagrangian,

$$
\mathcal{L}_{\text {inv }}=-\frac{1}{4} F_{\mu \nu} F_{\rho \sigma} g^{\mu \rho} g^{\sigma \nu} \sqrt{-g}=-\frac{1}{4} a^{D-4} F_{\mu \nu} F_{\rho \sigma} \eta^{\mu \rho} \eta^{\nu \sigma} .
$$

The wonderful simplicity of using known flat space results will not be preserved if one adds any multiple of the de Sitter invariant, Feynman gauge fixing functional,

$$
\mathcal{L}_{\mathrm{dS}}=-\frac{1}{2}\left(g^{\mu \nu} A_{\mu ; \nu}\right)^{2}=-\frac{1}{2} a^{D-4}\left(\eta^{\mu \nu} A_{\mu, \nu}-(D-2) H a A_{0}\right)^{2} .
$$

(A semicolon denotes covariant differentiation, whereas a comma stands for the ordinary derivative.) However, a very simple formalism results from replacing the factor of $(D-2)$ with $(D-4)$,

$$
\mathcal{L}_{\text {NdS }}=-\frac{1}{2} a^{D-4}\left(\eta^{\mu \nu} \partial_{\mu} A_{\nu}-(D-4) H a A_{0}\right)^{2} .
$$

With this gauge fixing functional, the propagator takes the form ${ }^{10,18}$

$$
i\left[{ }_{\mu} \Delta_{\nu}^{\mathrm{NdS}}\right](x ; z)=a_{x} a_{z} i \Delta_{B}(x ; z)\left(\eta_{\mu \nu}+\delta_{\mu}^{0} \delta_{\nu}^{0}\right)-a_{x} a_{z} i \Delta_{C}(x ; z) \delta_{\mu}^{0} \delta_{\nu}^{0},
$$

where the de Sitter invariant scalar propagators are

$$
\begin{aligned}
& i \Delta_{B}(x ; z) \equiv B(y(x ; z))=\frac{H^{D-2}}{(4 \pi)^{D / 2}} \frac{\Gamma(D-2)}{\Gamma\left(\frac{D}{2}\right)}{ }_{2} F_{1}\left(D-2,1 ; \frac{D}{2} ; 1-y\right), \\
& i \Delta_{C}(x ; z) \equiv C(y(x ; z))=\frac{H^{D-2}}{(4 \pi)^{D / 2}} \frac{\Gamma(D-3)}{\Gamma\left(\frac{D}{2}\right)}{ }_{2} F_{1}\left(D-3,2 ; \frac{D}{2} ; 1-y\right) .
\end{aligned}
$$

One nice thing about (68) is that its tensor factors are constants. Another is that each of the scalar propagators that multiply them consists of the conformal propagator plus a series of less singular terms, which vanish in $D=4$ dimensions, 


$$
\begin{gathered}
B(y)=\frac{H^{D-2}}{(4 \pi)^{D / 2}}\left\{\Gamma\left(\frac{D}{2}-1\right)\left(\frac{4}{y}\right)^{D / 2-1}+\sum_{n=0}^{\infty}\left[\frac{\Gamma\left(n+\frac{D}{2}\right)}{\Gamma(n+2)}\left(\frac{y}{4}\right)^{n-D / 2+2}-\frac{\Gamma(n+D-2)}{\Gamma\left(n+\frac{D}{2}\right)}\left(\frac{y}{4}\right)^{n}\right]\right\}, \\
C(y)=\frac{H^{D-2}}{(4 \pi)^{D / 2}}\left\{\Gamma\left(\frac{D}{2}-1\right)\left(\frac{4}{y}\right)^{D / 2-1}-\sum_{n=0}^{\infty}\left[\left(n-\frac{D}{2}+3\right) \frac{\Gamma\left(n+\frac{D}{2}-1\right)}{\Gamma(n+2)}\left(\frac{y}{4}\right)^{n-D / 2+2}\right.\right. \\
\left.\left.-(n+1) \frac{\Gamma(n+D-3)}{\Gamma\left(n+\frac{D}{2}\right)}\left(\frac{y}{4}\right)^{n}\right]\right\} .
\end{gathered}
$$

So the infinite series terms only need to be retained when they multiply a potentially divergent quantity. Because the higher values of $n$ vanish more and more rapidly at coincidence (that is, for $y=0$ ), only a finite number of these extra terms ever need to be included.

It is now time to make the field-dependent transformation to Lorentz gauge,

$$
A_{\mu}^{\prime}(x)=A_{\mu}(x)-\partial_{\mu} \theta[A](x) .
$$

This would be step (5) in the scheme of the previous section. The fact that $A_{\mu}^{\prime}$ obeys Lorentz gauge implies a differential equation for $\theta[A]$,

$$
\partial_{\mu}\left(\sqrt{-g} g^{\mu \nu} \partial_{\nu} \theta\right)=\partial_{\mu}\left(\sqrt{-g} g^{\mu \nu} A_{\nu}\right) .
$$

Of course, there are many solutions related to one another by homogeneous terms. Any choice of homogeneous term will enforce Lorentz gauge, whereas there can be at most one choice which gives a de Sitter invariant propagator. Because Sec. V is devoted to establishing de Sitter invariance and correspondence with the known solution, ${ }^{19}$ we postpone specification of the homogeneous term until then. For now, we express the solution in a general way,

$$
\bar{\theta}[A](x)=\int_{V} d^{D} x^{\prime} G\left(x ; x^{\prime}\right) \frac{\partial}{\partial x^{\prime \rho}}\left(\sqrt{-g\left(x^{\prime}\right)} g^{\rho \sigma}\left(x^{\prime}\right) A_{\sigma}\left(x^{\prime}\right)\right),
$$

where $G\left(x ; x^{\prime}\right)$ is some Green's function of the scalar d'Alembertian, which we specify in Sec. IV, and $V$ is some region of the manifold. The actual solution for $\theta[A](x)$ consists of (75) - with definite choices for $G\left(x ; x^{\prime}\right)$ and $V$-plus a definite homogeneous solution. For now, we study the field transformed with only $\bar{\theta}[A](x)$,

$$
\bar{A}_{\mu}(x) \equiv A_{\mu}(x)-\partial_{\mu} \bar{\theta}[A](x) .
$$

The transformed propagator is the vacuum expectation value of the $T^{*}$-ordered product of two $\bar{A}$ 's. Because $T^{*}$-ordering moves any derivatives outside the time-ordering symbol, we can express this as 


$$
\begin{aligned}
\left\langle\Omega\left|T^{*}\left[\bar{A}_{\mu}(x) \bar{A}_{\nu}(z)\right]\right| \Omega\right\rangle= & \left\langle\Omega\left|T\left[A_{\mu}(x) A_{\nu}(z)\right]\right| \Omega\right\rangle-\frac{\partial}{\partial x^{\mu}} \int_{V} d^{D} x^{\prime} G\left(x ; x^{\prime}\right) \times \frac{\partial}{\partial x^{\prime \rho}}\left[\sqrt{-g\left(x^{\prime}\right)} g^{\rho \sigma}\left(x^{\prime}\right)\right. \\
& \left.\times\left\langle\Omega\left|T\left[A_{\sigma}\left(x^{\prime}\right) A_{\nu}(z)\right]\right| \Omega\right\rangle\right]-\frac{\partial}{\partial z^{\nu}} \int_{V} d^{D} z^{\prime} G\left(z ; z^{\prime}\right) \times \frac{\partial}{\partial z^{\prime \alpha}}\left[\sqrt{-g\left(z^{\prime}\right)} g^{\alpha \beta}\left(z^{\prime}\right)\right. \\
& \left.\times\left\langle\Omega\left|T\left[A_{\mu}(x) A_{\beta}\left(z^{\prime}\right)\right]\right| \Omega\right\rangle\right]+\frac{\partial}{\partial x^{\mu}} \frac{\partial}{\partial z^{\nu}} \int_{V} d^{D} x^{\prime} G\left(x ; x^{\prime}\right) \int_{V} d^{D} z^{\prime} G\left(z ; z^{\prime}\right) \\
& \times \frac{\partial}{\partial x^{\prime \rho}} \frac{\partial}{\partial z^{\prime \alpha}}\left[\sqrt{-g\left(x^{\prime}\right)} g^{\rho \sigma}\left(x^{\prime}\right) \sqrt{-g\left(z^{\prime}\right)} g^{\alpha \beta}\left(z^{\prime}\right)\left\langle\Omega\left|T\left[A_{\sigma}\left(x^{\prime}\right) A_{\beta}\left(z^{\prime}\right)\right]\right| \Omega\right\rangle\right] .
\end{aligned}
$$

By substituting the noninvariant propagator $(68)$ and using the fact that $y(x ; z)$ depends on the spatial coordinates only through their difference, we can write the three differentiated, squarebracketed terms as

$$
\begin{aligned}
& \frac{\partial}{\partial x^{\prime \rho}}\left[\sqrt{-g\left(x^{\prime}\right)} g^{\rho \sigma}\left(x^{\prime}\right)\left\langle\Omega\left|T\left[A_{\sigma}\left(x^{\prime}\right) A_{\nu}(z)\right]\right| \Omega\right\rangle\right] \\
& =-\frac{\partial}{\partial z^{\nu}}\left[a_{x^{\prime}}^{D-1} a_{z} B\left(y\left(x^{\prime} ; z\right)\right)\right]+\delta_{\nu}^{0}\left\{\frac{\partial}{\partial z^{0}}\left[a_{x^{\prime}}^{D-1} a_{z} B\left(y\left(x^{\prime} ; z\right)\right)\right]+\frac{\partial}{\partial x^{\prime 0}}\left[a_{x^{\prime}}^{D-1} a_{z} C\left(y\left(x^{\prime} ; z\right)\right)\right]\right\} \\
& \begin{array}{c}
\frac{\partial}{\partial z^{\prime \alpha}}\left[\sqrt{-g\left(z^{\prime}\right)} g^{\alpha \beta}\left(z^{\prime}\right)\left\langle\Omega\left|T\left[A_{\mu}(x) A_{\beta}\left(z^{\prime}\right)\right]\right| \Omega\right\rangle\right] \\
=-\frac{\partial}{\partial x^{\mu}}\left[a_{x} a_{z^{\prime}}^{D-1} B\left(y\left(x ; z^{\prime}\right)\right)\right]+\delta_{\mu}^{0}\left\{\frac{\partial}{\partial x^{0}}\left[a_{x} a_{z^{\prime}}^{D-1} B\left(y\left(x ; z^{\prime}\right)\right)\right]+\frac{\partial}{\partial z^{\prime 0}}\left[a_{x} a_{z^{\prime}}^{D-1} C\left(y\left(x ; z^{\prime}\right)\right)\right]\right\} \\
\frac{\partial}{\partial x^{\prime \rho}} \frac{\partial}{\partial z^{\prime \alpha}}\left[\sqrt{-g\left(x^{\prime}\right)} g^{\rho \sigma}\left(x^{\prime}\right) \sqrt{-g\left(z^{\prime}\right)} g^{\alpha \beta}\left(z^{\prime}\right)\left\langle\Omega\left|T\left[A_{\sigma}\left(x^{\prime}\right) A_{\beta}\left(z^{\prime}\right)\right]\right| \Omega\right\rangle\right] \\
=\frac{\partial}{\partial x^{\prime i}} \frac{\partial}{\partial z^{\prime i}}\left[\left(a_{x^{\prime}} a_{z^{\prime}}\right)^{D-1} B\left(y\left(x^{\prime} ; z^{\prime}\right)\right)\right]-\frac{\partial}{\partial x^{\prime 0}} \frac{\partial}{\partial z^{\prime 0}}\left[\left(a_{x^{\prime}} a_{z^{\prime}}\right)^{D-1} C\left(y\left(x^{\prime} ; z^{\prime}\right)\right)\right]
\end{array}
\end{aligned}
$$

All of these suggest that we would do well to organize the transformed propagator into a doubly differentiated "integral term" and the remaining "other term,"

$$
\left\langle\Omega\left|T^{*}\left[\bar{A}_{\mu}(x) \bar{A}_{\nu}(z)\right]\right| \Omega\right\rangle=\frac{\partial}{\partial x^{\mu}} \frac{\partial}{\partial z^{\nu}} \overline{\mathcal{I}}(x ; z)+\left[{ }_{\mu} \overline{\mathcal{O}}_{\nu}\right](x ; z) .
$$

The integral term is 


$$
\begin{aligned}
\overline{\mathcal{I}}(x ; z) \equiv & a_{z} \int_{V} d^{D} x^{\prime} G\left(x ; x^{\prime}\right) a_{x^{\prime}}^{D-1} B\left(y\left(x^{\prime} ; z\right)\right)+a_{x} \int_{V} d^{D} z^{\prime} G\left(z ; z^{\prime}\right) a_{z^{\prime}}^{D-1} B\left(y\left(x ; z^{\prime}\right)\right) \\
& +\int_{V} d^{D} x^{\prime} G\left(x ; x^{\prime}\right) \int_{V} d^{D} z^{\prime} G\left(z ; z^{\prime}\right)\left\{\frac{\partial}{\partial x^{\prime i}} \frac{\partial}{\partial z^{\prime i}}\left[\left(a_{x^{\prime}} a_{z^{\prime}}\right)^{D-1} B\left(y\left(x^{\prime} ; z^{\prime}\right)\right)\right]\right. \\
& \left.-\frac{\partial}{\partial x^{\prime 0}} \frac{\partial}{\partial z^{\prime 0}}\left[\left(a_{x^{\prime}} a_{z^{\prime}}\right)^{D-1} C\left(y\left(x^{\prime} ; z^{\prime}\right)\right)\right]\right\} .
\end{aligned}
$$

Moreover, the other term contains everything else,

$$
\begin{aligned}
{\left[\overline{\mathcal{O}}_{\nu}\right](x ; z) \equiv } & a_{x} a_{z} B(y(x ; z))\left[\eta_{\mu \nu}+\delta_{\mu}^{0} \delta_{\nu}^{0}\right]-a_{x} a_{z} C(y(x ; z)) \delta_{\mu}^{0} \delta_{\nu}^{0}-\delta_{\nu}^{0} \frac{\partial}{\partial x^{\mu}} \int_{V} d^{D} x^{\prime} G\left(x ; x^{\prime}\right) \\
& \times\left\{\frac{\partial}{\partial z^{0}}\left[a_{x^{\prime}}^{D-1} a_{z} B\left(y\left(x^{\prime} ; z\right)\right)\right]+\frac{\partial}{\partial x^{\prime 0}}\left[a_{x^{\prime}}^{D-1} a_{z} C\left(y\left(x^{\prime} ; z\right)\right)\right]\right\}-\delta_{\mu}^{0} \frac{\partial}{\partial z^{\nu}} \int_{V} d^{D} z^{\prime} G\left(z ; z^{\prime}\right) \\
& \times\left\{\frac{\partial}{\partial x^{0}}\left[a_{x} a_{z^{\prime}}^{D-1} B\left(y\left(x ; z^{\prime}\right)\right)\right]+\frac{\partial}{\partial z^{\prime 0}}\left[a_{x} a_{z^{\prime}}^{D-1} C\left(y\left(x ; z^{\prime}\right)\right)\right]\right\}
\end{aligned}
$$

It remains to act the derivatives to simplify our expressions for $\overline{\mathcal{I}}(x ; z)$ and $\left[{ }_{\mu} \overline{\mathcal{O}}_{\nu}\right](x ; z)$. This is facilitated by some important identities obeyed by any function of $y(x ; z)$,

$$
\begin{gathered}
\square_{x} F(y)=\frac{i 4 \pi^{D / 2} \delta^{D}(x-z)}{\Gamma\left(\frac{D}{2}-1\right) H^{D-2} a^{D}} \times \operatorname{Res}[F]+H^{2}\left\{\left(4 y-y^{2}\right) F^{\prime \prime}+D(2-y) F^{\prime}\right\} \\
\frac{\partial^{2} F(y)}{\partial x^{0} \partial z^{0}}=\frac{i 4 \pi^{D / 2} \delta^{D}(x-z)}{\Gamma\left(\frac{D}{2}-1\right)(H a)^{D-2}} \times \operatorname{Res}[F]+a_{x} a_{z} H^{2}\left\{\left[8-\left(4 y-y^{2}\right)\right] F^{\prime \prime}-(2-y) F^{\prime}+\left(\frac{a_{x}}{a_{z}}+\frac{a_{z}}{a_{x}}\right)[-2(2\right. \\
\left.\left.-y) F^{\prime \prime}+2 F^{\prime}\right]\right\} \\
\frac{\partial^{2} F(y)}{\partial x^{i} \partial z^{i}}=a_{x} a_{z} H^{2}\left\{4(2-y) F^{\prime \prime}-2(D-1) F^{\prime}-4\left(\frac{a_{x}}{a_{z}}+\frac{a_{z}}{a_{x}}\right) F^{\prime \prime}\right\} \\
H\left[a_{x} \frac{\partial}{\partial z^{0}}+a_{z} \frac{\partial}{\partial x^{0}}\right] F(y)=a_{x} a_{z} H^{2}\left\{-2(2-y) F^{\prime}+2\left(\frac{a_{x}}{a_{z}}+\frac{a_{z}}{a_{x}}\right) F^{\prime}\right\}
\end{gathered}
$$

Here, $\square$ is the covariant scalar d'Alembertian and $\operatorname{Res}[F]$ is the coefficient of $y^{1-D / 2}$ in the Laurent expansion of $F(y)$. We shall also require some identities specific to $B(y)$ and $C(y)$,

$$
\begin{gathered}
\left(4 y-y^{2}\right) B^{\prime \prime}(y)+D(2-y) B^{\prime}(y)=(D-2) B(y), \\
\left(4 y-y^{2}\right) C^{\prime \prime}(y)+D(2-y) C^{\prime}(y)=2(D-3) C(y) .
\end{gathered}
$$

Also, there is a very useful relation between $B(y)$ and $C(y)$, 


$$
C(y)=\frac{1}{2}(2-y) B(y)+\frac{k}{D-3}, \quad \text { where } \quad k \equiv \frac{H^{D-2}}{(4 \pi)^{D / 2}} \frac{\Gamma(D-1)}{\Gamma\left(\frac{D}{2}\right)} .
$$

Note finally that substituting (90) into (89) and using (88) implies that

$$
\left(4 y-y^{2}\right) B^{\prime}(y)+(D-2)(2-y) B(y)=-2 k .
$$

It is best to start with the Other Term because it involves only first derivatives. The reduction is straightforward for the $B$ term,

$$
\begin{gathered}
\frac{\partial}{\partial z^{0}}\left[a_{x}^{D-1} a_{z} B(y(x ; z))\right]=a_{x}^{D-1} a_{z}\left[H a_{z} B+\frac{\partial y}{\partial z^{0}} B^{\prime}\right] \\
=a_{x}^{D} a_{z} H\left\{2 B^{\prime}+\frac{a_{z}}{a_{x}}\left[-(2-y) B^{\prime}+B\right]\right\} .
\end{gathered}
$$

We begin the same way with the $C$ term, but then use (90) to convert most of the $C$ 's to $B$ 's and simplify with (91),

$$
\begin{gathered}
\frac{\partial}{\partial x^{0}}\left[a_{x}^{D-1} a_{z} C(y(x ; z))\right]=a_{x}^{D-1} a_{z}\left[(D-1) H a_{x} C+\frac{\partial y}{\partial x^{0}} C^{\prime}\right] \\
=a_{x}^{D} a_{z} H\left\{-(2-y) C^{\prime}+(D-1) C+\frac{a_{z}}{a_{x}}\left[2 C^{\prime}\right]\right\} \\
=a_{x}^{D} a_{z} H\left\{2 C-\frac{1}{2}(2-y)^{2} B^{\prime}+\frac{1}{2}(D-2)(2-y) B+k+\frac{a_{z}}{a_{x}}\left[(2-y) B^{\prime}-B\right]\right\} \\
=a_{x}^{D} a_{z} H\left\{2 C-2 B^{\prime}+\frac{a_{z}}{a_{x}}\left[(2-y) B^{\prime}-B\right]\right\} .
\end{gathered}
$$

Hence, (93) and (97) almost completely cancel and our final result for the Other Term is

$$
\begin{aligned}
{\left[{ }_{\mu} \overline{\mathcal{O}}_{\nu}\right](x ; z) \equiv } & a_{x} a_{z} B(y(x ; z)) \eta_{\mu \nu}+a_{x} a_{z}\left\{\frac{1}{2} y(x ; z) B(y(x ; z))-\frac{k}{D-3}\right\} \delta_{\mu}^{0} \delta_{\nu}^{0} \\
& -2 H a_{z} \delta_{\nu}^{0} \frac{\partial}{\partial x^{\mu}} \int_{V} d^{D} x^{\prime} \sqrt{-g\left(x^{\prime}\right)} G\left(x ; x^{\prime}\right) C\left(y\left(x^{\prime} ; z\right)\right) \\
& -2 H a_{x} \delta_{\mu}^{0} \frac{\partial}{\partial z^{\nu}} \int_{V} d^{D} z^{\prime} \sqrt{-g\left(z^{\prime}\right)} G\left(z ; z^{\prime}\right) C\left(y\left(x ; z^{\prime}\right)\right) .
\end{aligned}
$$

The Integral Term $\overline{\mathcal{I}}(x ; z)$ involves second derivatives. We only need (86) to reduce the spatial case,

$$
\frac{\partial}{\partial x^{i}} \frac{\partial}{\partial z^{i}}\left[\left(a_{x} a_{z}\right)^{D-1} B(y(x ; z))\right]=H^{2}\left(a_{x} a_{z}\right)^{D}\left\{4(2-y) B^{\prime \prime}-2(D-1) B^{\prime}+\left(\frac{a_{x}}{a_{z}}+\frac{a_{z}}{a_{x}}\right)\left[-4 B^{\prime \prime}\right]\right\} .
$$

Reducing the temporal derivative term is more involved. We begin by passing the derivatives through the scale factors, then employ relations (85) and (87)), and convert most of the $C(y)$ 's to $B(y)$ using (90), eliminating second derivatives with (88) and (89) as needed. The result is 


$$
\begin{aligned}
& \frac{\partial}{\partial x^{0}} \frac{\partial}{\partial z^{0}}\left[\left(a_{x} a_{z}\right)^{D-1} C(y(x ; z))\right] \\
& \quad=\left(a_{x} a_{z}\right)^{D-1}\left\{\frac{\partial}{\partial x^{0}} \frac{\partial}{\partial z^{0}}+(D-1) H\left[a_{x} \frac{\partial}{\partial z^{0}}+a_{z} \frac{\partial}{\partial x^{0}}\right]+(D-1)^{2} a_{x} a_{z} H^{2}\right\} C(y(x ; z)) \\
& =i a_{x}^{D} \delta^{D}\left(x-z^{\prime}\right)+H^{2}\left(a_{x} a_{z}\right)^{D}\left\{4 C+4(2-y) B^{\prime \prime}-2(D+3) B^{\prime}+\left(\frac{a_{x}}{a_{z}}+\frac{a_{z}}{a_{x}}\right)\left[-4 B^{\prime \prime}+2(2-y) B^{\prime}\right.\right. \\
& -2 B]\} .
\end{aligned}
$$

Adding the two terms gives a compact form,

$$
\begin{aligned}
\frac{\partial}{\partial x^{i}} & \frac{\partial}{\partial z^{i}}\left[\left(a_{x} a_{z}\right)^{D-1} B(y)\right]-\frac{\partial}{\partial x^{0}} \frac{\partial}{\partial z^{0}}\left[\left(a_{x} a_{z}\right)^{D-1} C(y)\right] \\
\quad & =-i a_{x}^{D} \delta^{D}(x-z)+\left(a_{x} a_{z}\right)^{D} H^{2}\left\{-4 C+8 B^{\prime}+\left(\frac{a_{x}}{a_{z}}+\frac{a_{z}}{a_{x}}\right)\left[-2(2-y) B^{\prime}+2 B\right]\right\} .
\end{aligned}
$$

A further simplification can be effected by means of the identity,

$$
\left(a_{x} a_{z}\right)^{D} \square_{x}\left[\frac{a_{x}}{a_{z}} B(y)\right]=i a_{x}^{D} \delta^{D}(x-z)+\left(a_{x} a_{z}\right)^{D} H^{2}\left\{-4 B^{\prime}+\frac{a_{x}}{a_{z}}\left[2(2-y) B^{\prime}-2 B\right]\right\} .
$$

Using this and the result with $x^{\mu}$ and $z^{\mu}$ interchanged gives

$$
\begin{aligned}
& \frac{\partial}{\partial x^{i}} \frac{\partial}{\partial z^{i}}\left[\left(a_{x} a_{z}\right)^{D-1} B(y)\right]-\frac{\partial}{\partial x^{0}} \frac{\partial}{\partial z^{0}}\left[\left(a_{x} a_{z}\right)^{D-1} C(y)\right] \\
& \quad=i a_{x}^{D} \delta^{D}(x-z)+\left(a_{x} a_{z}\right)^{D}\left\{-4 H^{2} C-\square_{x}\left[\frac{a_{x}}{a_{z}} B\right]-\square_{z}\left[\frac{a_{z}}{a_{x}} B\right]\right\} .
\end{aligned}
$$

Our final result for the Integral Term involves the surface integral,

$$
\begin{gathered}
\mathcal{S}_{B}(x ; z) \equiv \frac{a_{x}}{a_{z}} B(y(x ; z))-\int_{V} d^{D} x^{\prime} \sqrt{-g\left(x^{\prime}\right)} G\left(x ; x^{\prime}\right) \square_{x^{\prime}}\left[\frac{a_{x^{\prime}}}{a_{z}} B\left(y\left(x^{\prime} ; z\right)\right)\right] \\
=\int_{\partial V} d^{D-1} x_{\mu}^{\prime} \sqrt{-g^{\prime}} g^{\prime \mu \nu}\left[\frac{a_{x^{\prime}}}{a_{z}} i \Delta_{B}\left(x^{\prime} ; z\right) \partial_{\nu}^{\prime} G\left(x ; x^{\prime}\right)-G\left(x ; x^{\prime}\right) \partial_{\nu}^{\prime}\left[\frac{a_{x^{\prime}}}{a_{z}} i \Delta_{B}\left(x^{\prime} ; z\right)\right]\right] .
\end{gathered}
$$

This function is obviously homogeneous; that is, $\square_{x}$ annihilates it. In Sec. IV we will show how to choose the homogeneous contribution to the full gauge parameter $\theta[A](x)$ so as to cancel it and similar terms. The final result for the Integral Term is

$$
\begin{aligned}
\overline{\mathcal{I}}(x ; z)= & \int_{V} d^{D} x^{\prime} \sqrt{-g\left(x^{\prime}\right)} G\left(x ; x^{\prime}\right) \mathcal{S}_{B}\left(z ; x^{\prime}\right)+\int_{V} d^{D} z^{\prime} \sqrt{-g\left(z^{\prime}\right)} G\left(z ; z^{\prime}\right) \mathcal{S}_{B}\left(x ; z^{\prime}\right) \\
& +i \int_{V} d^{D} x^{\prime} \sqrt{-g\left(x^{\prime}\right)} G\left(x ; x^{\prime}\right) G\left(z ; x^{\prime}\right) \\
& -4 H^{2} \int_{V} d^{D} x^{\prime} \sqrt{-g\left(x^{\prime}\right)} G\left(x ; x^{\prime}\right) \int_{V} d^{D} z^{\prime} \sqrt{-g\left(z^{\prime}\right)} G\left(z ; z^{\prime}\right) C\left(y\left(x^{\prime} ; z^{\prime}\right)\right) .
\end{aligned}
$$




\section{THE INVARIANT PROPAGATOR}

The purpose of this section is to show that the transformed propagator of the previous section agrees, up to surface terms, with the unique de Sitter invariant propagator, which was found by solving the propagator equation in Lorentz gauge. ${ }^{19}$ Of course, we begin by describing that solution. We then decompose it in analogy with the scheme (81) of the previous section, into an integral term and another term. At this stage, there is a digression to derive an identity for the convolution of scalar propagators. The section closes by applying this identity to demonstrate that the two propagators agree up to surface integrals.

The Lorentz gauge propagator equation has a unique de Sitter invariant solution which can expressed in terms of a function $\gamma(y),{ }^{19}$

$$
\begin{aligned}
i\left[{ }_{\mu} \Delta_{\nu}^{\mathrm{dS}}\right](x ; z)= & \frac{1}{4(D-1) H^{2}}\left\{\frac{\partial^{2} y(x ; z)}{\partial x^{\mu} \partial z^{\nu}}\left[-\left(4 y-y^{2}\right) \gamma^{\prime}-(D-1)(2-y) \gamma\right]+\frac{\partial y}{\partial x^{\mu}} \frac{\partial y}{\partial z^{\nu}}\left[(2-y) \gamma^{\prime}-(D\right.\right. \\
& -1) \gamma]\} .
\end{aligned}
$$

The function $\gamma(y)$ has a very complicated series expansion

$$
\begin{aligned}
\gamma(y)= & \frac{1}{2}\left(\frac{D-1}{D-3}\right) \frac{H^{D-2}}{(4 \pi)^{D / 2}}\left\{(D-3) \Gamma\left(\frac{D}{2}-1\right)\left(\frac{4}{y}\right)^{D / 2-1}+\sum_{n=0}^{\infty}\left[\frac{(n+1) \Gamma(n+D-1)}{\Gamma\left(n+\frac{D}{2}+1\right)} \times\left[\psi\left(2-\frac{D}{2}\right)\right.\right.\right. \\
& \left.-\psi\left(\frac{D}{2}-1\right)+\psi(n+D-1)-\psi(n+2)\right]\left(\frac{y}{4}\right)^{n}-\frac{\left(n-\frac{D}{2}+3\right) \Gamma\left(n+\frac{D}{2}+1\right)}{\Gamma(n+3)}\left[\psi\left(2-\frac{D}{2}\right)\right. \\
& \left.\left.\left.-\psi\left(\frac{D}{2}-1\right)+\psi\left(n+\frac{D}{2}+1\right)-\psi\left(n-\frac{D}{2}+4\right)\right]\left(\frac{y}{4}\right)^{n-D / 2+2}\right]\right\}
\end{aligned}
$$

Although it might seem unwieldy, this formalism has been used to perform several two loop computations in scalar QED..$^{22,23}$

The function $\gamma(y)$ obeys the second order differential equation,

$$
\left(4 y-y^{2}\right) \gamma^{\prime \prime}+(D+2)(2-y) \gamma^{\prime}-2(D-1) \gamma=2(D-1) B^{\prime}(y) .
$$

One consequence is

$$
\frac{\partial}{\partial y}\left[-\left(4 y-y^{2}\right) \gamma^{\prime}-(D-1)(2-y) \gamma+2(D-1) B\right]=(2-y) \gamma^{\prime}-(D-1) \gamma .
$$

Hence, we can decompose the invariant propagator in a form analogous to that of the transformed propagator (81),

$$
\begin{aligned}
i\left[{ }_{\mu} \Delta_{\nu}^{\mathrm{dS}}\right](x ; z)= & -\frac{1}{2 H^{2}} B(y(x ; z)) \frac{\partial^{2} y(x ; z)}{\partial x^{\mu} \partial z^{\nu}}+\frac{1}{4(D-1) H^{2}} \frac{\partial}{\partial x^{\mu}} \frac{\partial}{\partial z^{\nu}} I\left[-\left(4 y-y^{2}\right) \gamma^{\prime}-(D-1)(2-y) \gamma\right. \\
& +2(D-1) B],
\end{aligned}
$$

where the notation " $I[f]$ " of a function $f(y)$ stands for its indefinite integral,

$$
I[f](y) \equiv \int^{y} d y^{\prime} f\left(y^{\prime}\right) .
$$


At this point, it is useful to digress on the subject of scalar propagators. Three were introduced in Sec. III- $+i \times G(x ; z), i \Delta_{B}(x ; z)$, and $i \Delta_{C}(x ; z)$ - and it might seem that there is a bewildering variety of them, each with its own important special properties. However, a unified treatment can be given in terms of the equation,

$$
\sqrt{-g(x)}\left\{\square_{x}+\left[\nu^{2}-\left(\frac{D-1}{2}\right)^{2}\right] H^{2}\right\} i \Delta_{\nu}(x ; z)=i \delta^{D}(x-z) .
$$

The three propagators of Sec. III correspond to the following choices for $\nu$ :

$$
\begin{aligned}
& i \times G(x ; z) \Rightarrow \nu=\left(\frac{D-1}{2}\right), \\
& i \Delta_{B}(x ; z) \Rightarrow \nu=\left(\frac{D-3}{2}\right), \\
& i \Delta_{C}(x ; z) \Rightarrow \nu=\left(\frac{D-5}{2}\right) .
\end{aligned}
$$

For general $\nu$, the spatial plane wave mode functions corresponding to Bunch-Davies vacuum are

$$
u_{\nu}\left(x^{0}, k\right) \equiv \sqrt{\frac{\pi}{4 H}} a^{-(D-1) / 2} H_{\nu}^{(1)}\left(-k x^{0}\right) .
$$

When it exists, the Fourier mode sum for the propagator is ${ }^{24}$

$$
i \Delta_{\nu}(x ; z)=\int \frac{d^{D-1} k}{(2 \pi)^{D-1}} e^{i \vec{k} \cdot(\vec{x}-\vec{z})}\left\{\theta\left(x^{0}-z^{0}\right) u_{\nu}\left(x^{0}, k\right) u_{\nu}^{*}\left(z^{0}, k\right)+\theta\left(z^{0}-x^{0}\right) u_{\nu}^{*}\left(x^{0}, k\right) u_{\nu}\left(z^{0}, k\right)\right\}
$$

When this sum exists, the result is de Sitter invariant, ${ }^{25}$

$$
i \Delta_{\nu}(x ; z)=\frac{H^{D-2}}{(4 \pi)^{D / 2}} \frac{\Gamma\left(\frac{D-1}{2}+\nu\right) \Gamma\left(\frac{D-1}{2}-\nu\right)}{\Gamma\left(\frac{D}{2}\right)}{ }_{2} F_{1}\left(\frac{D-1}{2}+\nu, \frac{D-1}{2}-\nu ; \frac{D}{2} ; 1-\frac{y}{4}\right) .
$$

When the Fourier mode sum (119) is infrared divergent, it must be cut off either by making the mode functions less singular for superhorizon wavelengths ${ }^{26}$ or by working on a spatially compact manifold. ${ }^{27}$ Either procedure breaks de Sitter invariance. A special case of some importance to our discussion is $\nu=(D-1) / 2$, for which the result is ${ }^{28,24}$

$$
\nu=\left(\frac{D-1}{2}\right) \Rightarrow i \Delta_{A}(x ; z)=A(y(x ; z))+k \ln \left(a_{x} a_{z}\right)
$$

where the constant $k$ was defined in (90) and the function $A(y)$ has the expansion, 


$$
\begin{aligned}
A(y) \equiv & \frac{H^{D-2}}{(4 \pi)^{D / 2}}\left\{\frac{\Gamma\left(\frac{D}{2}\right)}{\frac{D}{2}-1}\left(\frac{4}{y}\right)^{D / 2-1}+\frac{\Gamma\left(\frac{D}{2}+1\right)}{\frac{D}{2}-2}\left(\frac{4}{y}\right)^{D / 2-2}-\pi \cot \left(\frac{\pi D}{2}\right) \frac{\Gamma(D-1)}{\Gamma\left(\frac{D}{2}\right)}\right. \\
& \left.+\sum_{n=1}^{\infty}\left[\frac{1}{n} \frac{\Gamma(n+D-1)}{\Gamma\left(n+\frac{D}{2}\right)}\left(\frac{y}{4}\right)^{n}-\frac{1}{n-\frac{D}{2}+2} \frac{\Gamma\left(n+\frac{D}{2}+1\right)}{\Gamma(n+2)}\left(\frac{y}{4}\right)^{n-D / 2+2}\right]\right\} .
\end{aligned}
$$

As with the expansions (71) and (72) for $B(y)$ and $C(y)$, the infinite series terms of $A(y)$ vanish for $D=4$, so they only need to be retained when multiplying a potentially divergent quantity and even then one only needs to include a handful of them. This makes loop computations manageable. For a massless, minimally coupled scalar with a quartic self-interaction, two loop results have been obtained for the expectation value of the stress tensor, ${ }^{28}$ for the scalar self-mass-squared ${ }^{29}$ and for the quantum-corrected mode functions. ${ }^{30}$ In Yukawa theory, it has been used to compute the expectation value of the coincident vertex function at two loop order, ${ }^{31}$ and it has been used for a variety of two loop computations in scalar QED. ${ }^{22,23}$ It should also be noted that the de Sitter breaking correction to $i \Delta_{A}(x ; z)$ in expression (121) can be derived from the infrared-truncated mode sum, ${ }^{24}$ and it serves to reproduce the classic and well known result for the coincidence limit of the propagator. ${ }^{32}$

The function $A(y)$ obeys a differential equation analogous to (88) and (89),

$$
\left(4 y-y^{2}\right) A^{\prime \prime}+D(2-y) A^{\prime}=(D-1) k .
$$

A number of identities relate the derivative of $A(y)$ to $B(y)$ and $C(y)$,

$$
\begin{gathered}
A^{\prime}=-\frac{1}{2}(D-3) B+C^{\prime}, \\
\left(4 y-y^{2}\right) A^{\prime}=-2(D-2) B-(2-y) k .
\end{gathered}
$$

It is also useful to note the result of acting the scalar d'Alembertian on a function of the scale factor,

$$
\square f(a)=-H^{2}\left[a^{2} f^{\prime \prime}(a)+D a f^{\prime}(a)\right] .
$$

Now consider Green's second identity for any two functions $F\left(x^{\prime}\right)$ and $G\left(x^{\prime}\right)$,

$$
\begin{aligned}
& F\left(x^{\prime}\right) \sqrt{-g\left(x^{\prime}\right)} \square^{\prime} G\left(x^{\prime}\right)-G\left(x^{\prime}\right) \sqrt{-g\left(x^{\prime}\right)} \square^{\prime} F\left(x^{\prime}\right) \\
& =\partial_{\mu}^{\prime}\left\{\sqrt{-g\left(x^{\prime}\right)} g^{\mu \nu}\left(x^{\prime}\right)\left[F\left(x^{\prime}\right) \partial_{\nu}^{\prime} G\left(x^{\prime}\right)-G\left(x^{\prime}\right) \partial_{\nu}^{\prime} F\left(x^{\prime}\right)\right]\right\} .
\end{aligned}
$$

We choose $G\left(x^{\prime}\right)$ to be any symmetric Green's function $G\left(x ; x^{\prime}\right)=G\left(x^{\prime} ; x\right)$,

$$
\sqrt{-g(x)} \square G\left(x ; x^{\prime}\right)=\delta^{D}\left(x-x^{\prime}\right) .
$$

We can obviously integrate (127) over any region $V$ with boundary $\partial V$ to conclude,

$$
\begin{aligned}
F(x)= & \int_{V} d^{D} x^{\prime} \sqrt{-g\left(x^{\prime}\right)} G\left(x ; x^{\prime}\right) \square_{x^{\prime}} F\left(x^{\prime}\right)+\int_{\partial V} d^{D-1} x_{\mu}^{\prime} \sqrt{-g^{\prime}} g^{\prime \mu \nu}\left[F\left(x^{\prime}\right) \partial_{\nu}^{\prime} G\left(x ; x^{\prime}\right)\right. \\
& \left.-G\left(x ; x^{\prime}\right) \partial_{\nu}^{\prime} F\left(x^{\prime}\right)\right] .
\end{aligned}
$$

Relation (129) is true for any Green's function so we are free to use the $A$-type propagator, $G\left(x ; x^{\prime}\right)=-i \times i \Delta_{A}\left(x ; x^{\prime}\right)$. Relation (129) is also valid for any function $F(x)$ so we are free to make the choice, 


$$
F(x) \rightarrow \frac{i \Delta_{\nu}(x ; z)-i \Delta_{A}(x ; z)}{\left[\left(\frac{D-1}{2}\right)^{2}-\nu^{2}\right] H^{2}}
$$

The surface terms involving $i \Delta_{A}$ obviously cancel so the result is

$$
\begin{aligned}
-i \int_{V} & d^{D} x^{\prime} \sqrt{-g\left(x^{\prime}\right)} i \Delta_{A}\left(x ; x^{\prime}\right) i \Delta_{\nu}\left(x^{\prime} ; z\right) \\
= & \frac{i \Delta_{\nu}(x ; z)-i \Delta_{A}(x ; z)}{\left[\left(\frac{D-1}{2}\right)^{2}-\nu^{2}\right] H^{2}} \\
& +i \int_{\partial V} d^{D-1} x_{\rho}^{\prime} \sqrt{-g^{\prime}} g^{\prime \rho \sigma}\left[\frac{i \Delta_{\nu}\left(x^{\prime} ; z\right) \partial_{\sigma}^{\prime} i \Delta_{A}\left(x ; x^{\prime}\right)-i \Delta_{A}\left(x ; x^{\prime}\right) \partial_{\sigma^{\prime}}^{\prime} i \Delta_{\nu}\left(x^{\prime} ; z\right)}{\left[\left(\frac{D-1}{2}\right)^{2}-\nu^{2}\right] H^{2}}\right] .
\end{aligned}
$$

We call (131) the "convolution identity."

Choosing $\nu=(D-5) / 2$ in the convolution identity (131) gives us a relation for the $C$-type propagator $C(y)$,

$$
-i \int_{V} d^{D} x^{\prime} \sqrt{-g\left(x^{\prime}\right)} i \Delta_{A}\left(x ; x^{\prime}\right) i \Delta_{C}\left(x^{\prime} ; z\right)=\frac{i \Delta_{C}(x ; z)-i \Delta_{A}(x ; z)-\mathcal{S}_{C}(x ; z)}{2(D-3) H^{2}},
$$

where the surface term is

$$
\mathcal{S}_{C}(x ; z) \equiv \int_{\partial V} d^{D-1} x_{\rho}^{\prime} \sqrt{-g^{\prime}} g^{\prime \rho \sigma}\left[i \Delta_{C}\left(x^{\prime} ; z\right) \partial_{\sigma}^{\prime} G\left(x ; x^{\prime}\right)-G\left(x ; x^{\prime}\right) \partial_{\sigma}^{\prime} i \Delta_{C}\left(x^{\prime} ; z\right)\right]
$$

We now substitute (132) in our result (98) for the Other Term in Sec. IV,

$$
\begin{aligned}
{\left[_{\mu} \overline{\mathcal{O}}_{\nu}\right](x ; z)=} & a_{x} a_{z} B(y(x ; z)) \eta_{\mu \nu}+a_{x} a_{z}\left\{\frac{1}{2} y(x ; z) B(y(x ; z))-\frac{k}{D-3}\right\} \delta_{\mu}^{0} \delta_{\nu}^{0}-2 H\left[a_{z} \delta_{\nu}^{0} \frac{\partial}{\partial x^{\mu}}\right. \\
& \left.+a_{x} \delta_{\mu}^{0} \frac{\partial}{\partial z^{\nu}}\right]\left\{\frac{i \Delta_{C}(x ; z)-i \Delta_{A}(x ; z)}{2(D-3) H^{2}}\right\}+\frac{a_{z} \delta_{\nu}^{0}}{(D-3) H} \frac{\partial \mathcal{S}_{C}(x ; z)}{\partial x^{\mu}}+\frac{a_{x} \delta_{\mu}^{0}}{(D-3) H} \frac{\partial \mathcal{S}_{C}(z ; x)}{\partial z^{\nu}} .
\end{aligned}
$$

The derivative is easy to simplify using (124),

$$
\begin{gathered}
-2 H a_{z} \delta_{\nu}^{0} \frac{\partial}{\partial x^{\mu}}\left\{\frac{i \Delta_{C}(x ; z)-i \Delta_{A}(x ; z)}{2(D-3) H^{2}}\right\}=-\frac{a_{z} \delta_{\nu}^{0}}{(D-3) H} \frac{\partial y}{\partial x^{\mu}}\left(C^{\prime}-A^{\prime}\right)+\frac{k}{D-3} \delta_{\mu}^{0} \delta_{\nu}^{0} a_{x} a_{z} \\
=a_{x} a_{z}\left\{-a_{z} H \Delta x_{\mu} \delta_{\nu}^{0} B-\frac{1}{2} y B \delta_{\mu}^{0} \delta_{\nu}^{0}+\frac{k}{D-3} \delta_{\mu}^{0} \delta_{\nu}^{0}\right\} .
\end{gathered}
$$

Combining everything results in an expression for the Other Term, which is almost de Sitter invariant, modulo the surface terms, 


$$
\begin{aligned}
{\left[_{\mu} \overline{\mathcal{O}}_{\nu}\right](x ; z)=} & a_{x} a_{z}\left\{-\frac{1}{2} y \delta_{\mu}^{0} \delta_{\nu}^{0}-a_{z} H \Delta x_{\mu} \delta_{\nu}^{0}+a_{x} \delta_{\mu}^{0} H \Delta x_{\nu}+\eta_{\mu \nu}\right\} B+\frac{k}{D-3} a_{x} a_{z} \delta_{\mu}^{0} \delta_{\nu}^{0} \\
& +\frac{a_{z} \delta_{\nu}^{0}}{(D-3) H} \frac{\partial \mathcal{S}_{C}(x ; z)}{\partial x^{\mu}}+\frac{a_{x} \delta_{\mu}^{0}}{(D-3) H} \frac{\partial \mathcal{S}_{C}(z ; x)}{\partial z^{\nu}} \\
=- & \frac{\partial^{2} y(x ; z)}{\partial x^{\mu} \partial z^{\nu}} \frac{B}{2 H^{2}}+\frac{k a_{x} a_{z} \delta_{\mu}^{0} \delta_{\nu}^{0}}{D-3}+\frac{a_{z} \delta_{\nu}^{0}}{D-3} \frac{\partial \mathcal{S}_{C}(x ; z)}{\partial H x^{\mu}}+\frac{a_{x} \delta_{\mu}^{0}}{D-3} \frac{\partial \mathcal{S}_{C}(z ; x)}{\partial H z^{\nu}} .
\end{aligned}
$$

The first term on the right hand side of (138) agrees with the first term in our decomposition (112) for the invariant propagator. We must obviously choose the homogeneous contributions to the gauge parameter $\theta[A](x)$ so as to cancel the surface terms in (138). That leaves the term proportional to $k$, which relation (126) allows us to recognize as a potential part of the Integral Term,

$$
\frac{k a_{x} a_{z} \delta_{\mu}^{0} \delta_{v}^{0}}{D-3}=\frac{\partial}{\partial x^{\mu}} \frac{\partial}{\partial z^{\nu}}\left\{\frac{k \ln ^{2}\left(a_{x} a_{z}\right)}{2(D-3) H^{2}}+\text { const } \times \ln \left(a_{x} a_{z}\right)\right\} .
$$

We will presently see that precisely the bracketed expression is needed to make $\overline{\mathcal{I}}(x ; z)$ de Sitter invariant up to surface terms.

A matter of great importance for us is what the convolution identity (131) gives when the index $\nu$ is chosen to be $(D-1) / 2$, corresponding to the $A$-type propagator. The term on the right hand side obviously gives a derivative with respect to the index $\nu$,

$$
\begin{gathered}
\lim _{\nu \rightarrow(D-1 / 2)}\left[\left[\frac{i \Delta_{\nu}(x ; z)-i \Delta_{A}(x ; z)}{\left[\left(\frac{D-1}{2}\right)^{2}-\nu^{2}\right] H^{2}}\right]=-\left.\frac{\frac{\partial}{\partial \nu} i \Delta_{\nu}(x ; z)}{(D-1) H^{2}}\right|_{\nu=((D-1) / 2)}\right. \\
\equiv-\frac{i \Delta_{A^{\prime}}(x ; z)}{(D-1) H^{2}}
\end{gathered}
$$

Hence, the convolution of two A-type propagators gives

$$
-i \int_{V} d^{D} x^{\prime} \sqrt{-g\left(x^{\prime}\right)} i \Delta_{A}\left(x ; x^{\prime}\right) i \Delta_{A}\left(x^{\prime} ; z\right)=-\frac{i \Delta_{A^{\prime}}(x ; z)}{(D-1) H^{2}}+\frac{\mathcal{S}_{A}(x ; z)}{(D-1) H^{2}},
$$

where the surface term is

$$
\mathcal{S}_{A}(x ; z) \equiv \int_{\partial V} d^{D-1} x_{\rho}^{\prime} \sqrt{-g^{\prime}} g^{\prime \rho \sigma}\left[i \Delta_{A^{\prime}}\left(x^{\prime} ; z\right) \partial_{\sigma}^{\prime} G\left(x ; x^{\prime}\right)-G\left(x ; x^{\prime}\right) \partial_{\sigma}^{\prime} i \Delta_{A^{\prime}}\left(x^{\prime} ; z\right)\right] .
$$

Like the $A$ propagator, the $A^{\prime}$ propagator breaks de Sitter invariance. The simplest way to see this is by differentiating relation (114) with respect to $\nu$ and then setting $\nu=(D-1) / 2$,

$$
\begin{gathered}
0=\frac{\partial}{\partial \nu}\left\{\left[\square_{x}+\left[\nu^{2}-\left(\frac{D-1}{2}\right)^{2}\right] H^{2}\right] i \Delta_{\nu}(x ; z)\right\}_{\nu=((D-1) / 2)} \\
=\square_{x} i \Delta_{A^{\prime}}(x ; z)+(D-1) H^{2} i \Delta_{A}(x ; z) .
\end{gathered}
$$

Because $i \Delta_{A}(x ; z)=A(y)+k \ln \left(a_{x} a_{z}\right)$ has a de Sitter breaking term, it is clear that $i \Delta_{A^{\prime}}(x ; z)$ must as well. From relation (126), we infer that 


$$
i \Delta_{A^{\prime}}(x ; z)=\mathcal{A}(y(x ; z))+\text { const } \times \ln \left(a_{x} a_{z}\right)+\frac{1}{2} k \ln ^{2}\left(a_{x} a_{z}\right) .
$$

We do not require the coefficient of the $\ln \left(a_{x} a_{z}\right)$ term but the series expansion for $\mathcal{A}(y)$ is

$$
\begin{aligned}
\mathcal{A}(y)= & \frac{H^{D-2}}{(4 \pi)^{D / 2}}\left(\frac{4}{y}\right)^{D / 2-2} \times 2\left(\frac{D-1}{D-4}\right) \Gamma\left(\frac{D}{2}-1\right)-\frac{H^{D-2}}{(4 \pi)^{D / 2}} \sum_{n=0}^{\infty}\left\{\frac{\left(\frac{1}{4} y\right)^{n-D / 2+3}}{n-\frac{D}{2}+3}\right. \\
& \times \frac{\Gamma\left(n+\frac{D}{2}+2\right)}{(n+2) !}\left[\psi\left(2-\frac{D}{2}\right)-\psi\left(\frac{D}{2}-1\right)+\psi\left(n+\frac{D}{2}+2\right)-\psi\left(n-\frac{D}{2}+3\right)\right]-\frac{\left(\frac{1}{4} y\right)^{n+1}}{n+1} \\
& \left.\times \frac{\Gamma(n+D)}{\Gamma\left(n+\frac{D}{2}+1\right)}\left[\psi\left(2-\frac{D}{2}\right)-\psi\left(\frac{D}{2}-1\right)+\psi(n+D)-\psi(n+1)\right]\right\} .
\end{aligned}
$$

One can hardly fail to notice the similarities in the series expansion (109) for $\gamma(y)$ and the expansion (147) we have just given for the de Sitter invariant part of the $A^{\prime}$ propagator. The relation between them is

$$
\mathcal{A}(y)=\frac{1}{4}\left(\frac{D-3}{D-1}\right) I\left[\left(4 y-y^{2}\right) \gamma^{\prime}+(D-1)(2-y) \gamma\right]-\frac{1}{2}(D-2) I[B]+\text { const. }
$$

It is tedious but straightforward to check (148) using the series expansions but a simpler way of recognizing it is to act the scalar d'Alembertian on both sides. In view of (145), the left hand side gives

$$
\frac{\square}{H^{2}} \mathcal{A}(y)=-(D-1) A(y)+\text { const. }
$$

To compute the right hand side, we need the lemma,

$$
(2-y)\left(4 y-y^{2}\right) \gamma^{\prime}+\left(4 y-y^{2}\right) \gamma+D(2-y)^{2} \gamma=2(D-1) I\left[(2-y) B^{\prime}\right]+\text { const. }
$$

This follows from differentiation with respect to $y$ and using Eq. (110) for $\gamma(y)$. Now act $\square / H^{2}$ on the first term on the right hand side of (148), then use the $\gamma$ Eq. (110), and finally relations (91) and (150),

$$
\begin{gathered}
\frac{\square}{H^{2}} I\left[\left(4 y-y^{2}\right) \gamma^{\prime}+(D-1)(2-y) \gamma\right]=\left(4 y-y^{2}\right)\left[\left(4 y-y^{2}\right) \gamma^{\prime \prime}+(D+1)(2-y) \gamma^{\prime}-(D-1) \gamma\right]+D(2 \\
-y)\left[\left(4 y-y^{2}\right) \gamma^{\prime}+(D-1)(2-y) \gamma\right] \\
=(D-1)\left\{(2-y)\left(4 y-y^{2}\right) \gamma^{\prime}+\left(4 y-y^{2}\right) \gamma+D(2-y)^{2} \gamma+2\left(4 y-y^{2}\right) B^{\prime}\right\} \\
=2(D-1)\left\{(D-1) I\left[(2-y) B^{\prime}\right]-(D-2)(2-y) B^{\prime}+\text { const }\right\} .
\end{gathered}
$$

Acting on the right hand side of (148) and using identities (91), (90), and (124) proves the relation,

$$
\begin{aligned}
\frac{\square}{H^{2}} & \left\{\frac{1}{4}\left(\frac{D-3}{D-1}\right) I\left[\left(4 y-y^{2}\right) \gamma^{\prime}+(D-1)(2-y) \gamma\right]-\frac{1}{2}(D-2) I[B]\right\}=\frac{1}{2}(D-3)(D-1) I\left[(2-y) B^{\prime}\right] \\
& -\frac{1}{2}(D-3)(D-2)(2-y) B-(D-2)(2-y) B+\mathrm{const}
\end{aligned}
$$




$$
\begin{gathered}
=\frac{1}{2}(D-3)(D-1) I[B]-\frac{1}{2}(D-1)(2-y) B+\text { const } \\
=-(D-1) A+\text { const. }
\end{gathered}
$$

The point of enduring all this analysis is that we can now recognize the Integral Term of the invariant propagator (112) as a collection of propagators plus the $\ln ^{2}\left(a_{x} a_{z}\right)$ term of expression (139),

$$
\begin{gathered}
\mathcal{I}(x ; z) \equiv \frac{1}{4(D-1) H^{2}} I\left[-\left(4 y-y^{2}\right) \gamma^{\prime}-(D-1)(2-y) \gamma+2(D-1) B\right] \\
=-\frac{\mathcal{A}}{(D-3) H^{2}}-\left[\frac{C-A}{(D-3)^{2} H^{2}}\right]+\text { const } \\
=-\frac{i \Delta_{A^{\prime}}(x ; z)}{(D-3) H^{2}}-\left[\frac{i \Delta_{C}(x ; z)-i \Delta_{A}(x ; z)}{(D-3)^{2} H^{2}}\right]+\frac{k \ln ^{2}\left(a_{x} a_{z}\right)}{2(D-3) H^{2}}+\text { const } \times \ln \left(a_{x} a_{z}\right)+\text { const. }
\end{gathered}
$$

Note that the two unknown constants are irrelevant because they drop out when one differentiates with respect to $x^{\mu}$ and $z^{\nu}$ to get the propagator.

We can make contact between the Integral Term (159) of the invariant propagator and the Integral Term (107) of the transformed propagator by expressing the propagators as convolution integrals,

$$
\begin{aligned}
& -\frac{i \Delta_{A^{\prime}}(x ; z)}{(D-3) H^{2}}=\left(\frac{D-1}{D-3}\right) \int_{V} d^{D} x^{\prime} \sqrt{-g\left(x^{\prime}\right)} G\left(x ; x^{\prime}\right) i \Delta_{A}\left(x^{\prime} ; z\right)-\frac{\mathcal{S}_{A}(x ; z)}{(D-3) H^{2}}, \\
& -\left[\frac{i \Delta_{C}(x ; z)-i \Delta_{A}(x ; z)}{(D-3)^{2} H^{2}}\right]=-\int_{V} d^{D} x^{\prime} \sqrt{-g\left(x^{\prime}\right)} G\left(x ; x^{\prime}\right) \frac{i \Delta_{C}\left(x^{\prime} ; z\right)}{D-3}-\frac{\mathcal{S}_{C}(x ; z)}{2(D-3)^{2} H^{2}} \\
& -\int_{V} d^{D} z^{\prime} \sqrt{-g\left(z^{\prime}\right)} G\left(z ; z^{\prime}\right) \frac{i \Delta_{C}\left(x ; z^{\prime}\right)}{D-3}-\frac{\mathcal{S}_{C}(z ; x)}{2(D-3)^{2} H^{2}} .
\end{aligned}
$$

Now break up the prefactor of (160) as

$$
\left(\frac{D-1}{D-3}\right)=1+\frac{1}{D-3}+\frac{1}{D-3}
$$

and combine the convolutions multiplying the last two factors with the convolutions of (161) to produce the combination $i \Delta_{C}-i \Delta_{A}$ that can be recognized as another convolution,

$$
\begin{aligned}
-\frac{i \Delta_{A^{\prime}}(x ; z)}{(D-3) H^{2}}-\left[\frac{i \Delta_{C}(x ; z)-i \Delta_{A}(x ; z)}{(D-3)^{2} H^{2}}\right]= & -i \int_{V} d^{D} x^{\prime} \sqrt{-g\left(x^{\prime}\right)} i \Delta_{A}\left(x ; x^{\prime}\right) i \Delta_{A}\left(x^{\prime} ; z\right) \\
& +i \int_{V} d^{D} x^{\prime} \sqrt{-g\left(x^{\prime}\right)} i \Delta_{A}\left(x ; x^{\prime}\right)\left[\frac{i \Delta_{C}\left(z ; x^{\prime}\right)-i \Delta_{A}\left(z ; x^{\prime}\right)}{D-3}\right] \\
& +i \int_{V} d^{D} z^{\prime} \sqrt{-g\left(z^{\prime}\right) i \Delta_{A}}\left(z ; z^{\prime}\right)\left[\frac{i \Delta_{C}\left(x ; z^{\prime}\right)-i \Delta_{A}\left(x ; z^{\prime}\right)}{D-3}\right] \\
& -\frac{\mathcal{S}_{A}(x ; z)}{(D-3) H^{2}}-\frac{\mathcal{S}_{C}(x ; z)}{2(D-3)^{2} H^{2}}-\frac{\mathcal{S}_{C}(z ; x)}{2(D-3)^{2} H^{2}}
\end{aligned}
$$




$$
\begin{aligned}
= & -i \int_{V} d^{D} x^{\prime} \sqrt{-g\left(x^{\prime}\right)} i \Delta_{A}\left(x ; x^{\prime}\right) i \Delta_{A}\left(x^{\prime} ; z\right) \\
& +4 H^{2} \int_{V} d^{D} x^{\prime} \sqrt{-g\left(x^{\prime}\right)} i \Delta_{A}\left(x ; x^{\prime}\right) \int_{V} d^{D} z^{\prime} \sqrt{-g\left(z^{\prime}\right)} i \Delta_{A}\left(z ; z^{\prime}\right) i \Delta_{C}\left(x^{\prime} ; z^{\prime}\right) \\
& -\int_{V} d^{D} x^{\prime} \sqrt{-g\left(x^{\prime}\right)} G\left(x ; x^{\prime}\right) \frac{\mathcal{S}_{C}\left(z ; x^{\prime}\right)}{D-3}-\int_{V} d^{D} z^{\prime} \sqrt{-g\left(z^{\prime}\right)} G\left(z ; z^{\prime}\right) \frac{\mathcal{S}_{C}\left(x ; z^{\prime}\right)}{D-3}-\frac{\mathcal{S}_{A}(x ; z)}{(D-3) H^{2}} \\
& -\frac{\mathcal{S}_{C}(x ; z)}{2(D-3)^{2} H^{2}}-\frac{\mathcal{S}_{C}(z ; x)}{2(D-3)^{2} H^{2}} .
\end{aligned}
$$

We obtain the desired relation by adding the Integral Term (107) of the transformed propagator to the $\ln ^{2}\left(a_{x} a_{z}\right)$ contribution (139) from the Other Term (and some pieces which drop out when differentiated by $x^{\mu}$ and $\left.z^{\nu}\right)$,

$$
\begin{aligned}
\overline{\mathcal{I}}(x ; z) & +\frac{k \ln ^{2}\left(a_{x} a_{z}\right)}{2(D-3) H^{2}}+\text { const } \times \ln \left(a_{x} a_{z}\right)+\text { const } \\
= & \mathcal{I}(x ; z)+\frac{\mathcal{S}_{A}(x ; z)}{(D-3) H^{2}}+\left[\frac{\mathcal{S}_{C}(x ; z)+\mathcal{S}_{C}(z ; x)}{2(D-3)^{2} H^{2}}\right]+\int_{V} d^{D} x^{\prime} \sqrt{-g\left(x^{\prime}\right)} G\left(x ; x^{\prime}\right)\left[\mathcal{S}_{B}\left(z ; x^{\prime}\right)\right. \\
& \left.+\frac{\mathcal{S}_{C}\left(z ; x^{\prime}\right)}{D-3}\right]+\int_{V} d^{D} z^{\prime} \sqrt{-g\left(z^{\prime}\right)} G\left(z ; z^{\prime}\right)\left[\mathcal{S}_{B}\left(x ; z^{\prime}\right)+\frac{\mathcal{S}_{C}\left(x ; z^{\prime}\right)}{D-3}\right]
\end{aligned}
$$

\section{DETERMINING THE HOMOGENEOUS PART}

The purpose of this section is to show that we can make the transformed propagator agree with the invariant one by correctly choosing the homogeneous part of the full gauge parameter $\theta[A](x)$. We begin by summarizing the relevant results of the previous two sections concerning the gauge parameter $\bar{\theta}[A](x)$, given in (75), which enforces Lorentz gauge but not de Sitter invariance. The resulting propagator agrees with the invariant one (108) and (109) up to three surface terms which we denote as " $A$-type," " $B$-type," and " $C$-type" according to the mode functions which they involve. We then exhibit a homogeneous gauge parameter $\Delta \theta[A](x)$, depending upon $A_{0}$, which can be used to absorb the $B$-type and $C$-type surface terms. The section closes by deriving a homogeneous gauge parameter $\delta \theta[A](x)$, depending on $A_{i}$, which absorbs the $A$-type surface terms and results in complete agreement with the invariant propagator.

\section{A. Summary of previous results}

Our goal is to construct a functional change in variables that is also a gauge transformation,

$$
A_{\mu}^{\prime}(x)=A_{\mu}(x)-\partial_{\mu} \theta[A](x) .
$$

We want the field-dependent gauge parameter $\theta[A](x)$ to do two things:

(1) make the field $A_{\mu}^{\prime}(x)$ obey Lorentz gauge and

(2) make the propagator associated with $A_{\mu}^{\prime}(x)$ agree with the unique, de Sitter invariant solution of the Lorentz gauge propagator equation. ${ }^{19}$

The first condition implies a second order differential equation for $\theta[A](x)$,

$$
\sqrt{-g} \square \theta=\partial_{\mu}\left[\sqrt{-g} g^{\mu \nu} A_{\nu}\right] .
$$

Of course, this only defines $\theta[A](x)$ up to a term which is annihilated by the scalar d'Alembertian. Because propagators obey Feynman boundary conditions, we took the inhomogeneous solution to 
be the convolution of $-i$ times the scalar propagator $\left(G(x ; z) \equiv-i \times i \Delta_{A}(x ; z)\right)$ with the right hand side of (167),

$$
\bar{\theta}[A](x) \equiv \int_{V} d^{D} x^{\prime} G\left(x ; x^{\prime}\right) \frac{\partial}{\partial x^{\prime \rho}}\left[\sqrt{-g\left(x^{\prime}\right)} g^{\rho \sigma}\left(x^{\prime}\right) A_{\sigma}\left(x^{\prime}\right)\right] .
$$

The result of just performing this transformation defines a field,

$$
\bar{A}_{\mu}(x) \equiv A_{\mu}(x)-\partial_{\mu} \bar{\theta}[A](x),
$$

which obeys the Lorentz gauge condition but whose propagator does not quite agree with the invariant one.

We decomposed the propagator of $\bar{A}_{\mu}(x)$ into the double gradient of an Integral Term (107) and an Other Term (98),

$$
\left\langle\Omega\left|T^{*}\left[\bar{A}_{\mu}(x) \bar{A}_{\nu}(z)\right]\right| \Omega\right\rangle=\frac{\partial}{\partial x^{\mu}} \frac{\partial}{\partial z^{\nu}} \overline{\mathcal{I}}(x ; z)+\left[{ }_{\mu} \overline{\mathcal{O}}_{\nu}\right](x ; z) .
$$

The invariant propagator can be broken up in similar fashion (112),

$$
i\left[{ }_{\mu} \Delta_{\nu}^{\mathrm{dS}}\right](x ; z)=\frac{\partial}{\partial x^{\mu}} \frac{\partial}{\partial z^{\nu}} \mathcal{I}(x ; z)-\frac{1}{2 H^{2}} B(y(x ; z)) \frac{\partial^{2} y(x ; z)}{\partial x^{\mu} \partial z^{\nu}} .
$$

It is desirable to shift the double gradient of a spatially constant term,

$$
\Delta \overline{\mathcal{I}}(x ; z) \equiv \frac{k \ln ^{2}\left(a_{x} a_{z}\right)}{2(D-3)}+\mathrm{const} \times \ln \left(a_{x} a_{z}\right)+\mathrm{const}
$$

from $\left[{ }_{\mu} \overline{\mathcal{O}}_{\nu}\right](x ; z)$ to $\overline{\mathcal{I}}(x ; z)$. When this is done, the difference between what we want the full transformation to produce and what the $\bar{\theta}[A](x)$ transformation actually gives is

$$
\begin{aligned}
{\left[{ }_{\mu} \mathcal{O}_{\nu}\right]-\left[\overline{\mathcal{O}}_{\nu}\right] } & +\frac{\partial^{2} \Delta \overline{\mathcal{I}}}{\partial x^{\mu} \partial z^{\nu}}=-\frac{a_{z} \delta_{\nu}^{0}}{D-3} \frac{\partial \mathcal{S}_{C}(x ; z)}{\partial H x^{\mu}}-\frac{a_{x} \delta_{\mu}^{0}}{D-3} \frac{\partial \mathcal{S}_{C}(z ; x)}{\partial H z^{\nu}} \\
\mathcal{I}(x ; z)-\overline{\mathcal{I}}(x ; z)-\Delta \overline{\mathcal{I}}(x ; z)= & -\frac{\mathcal{S}_{A}(x ; z)}{(D-3) H^{2}}-\left[\frac{\mathcal{S}_{C}(x ; z)+\mathcal{S}_{C}(z ; x)}{2(D-3)^{2} H^{2}}\right]-\int_{V} d^{D} x^{\prime} \sqrt{-g\left(x^{\prime}\right)} G\left(x ; x^{\prime}\right) \\
& \times\left[\mathcal{S}_{B}\left(z ; x^{\prime}\right)+\frac{\mathcal{S}_{C}\left(z ; x^{\prime}\right)}{D-3}\right]-\int_{V} d^{D} z^{\prime} \sqrt{-g\left(z^{\prime}\right)} G\left(z ; z^{\prime}\right)\left[\mathcal{S}_{B}\left(x ; z^{\prime}\right)\right. \\
& \left.+\frac{\mathcal{S}_{C}\left(x ; z^{\prime}\right)}{D-3}\right] .
\end{aligned}
$$

Each of the surface integrals, $\mathcal{S}_{F}(x ; z)$, consists of a Dirichlet and a Neumann contribution,

$$
\mathcal{S}_{F}(x ; z) \equiv \int_{\partial V} d^{D-1} x_{\rho}^{\prime} \sqrt{-g^{\prime}} g^{\prime \rho \sigma}\left[F\left(x^{\prime} ; z\right) \partial_{\sigma}^{\prime} G\left(x ; x^{\prime}\right)-G\left(x ; x^{\prime}\right) \partial_{\sigma}^{\prime} F\left(x^{\prime} ; z\right)\right] .
$$

The functions $F(x ; z)$ associated with the three integrals are

$$
\begin{gathered}
\mathcal{S}_{A}(x ; z) \Rightarrow F\left(x^{\prime} ; z\right)=i \Delta_{A^{\prime}}\left(x^{\prime} ; z\right), \\
\mathcal{S}_{B}(x ; z) \Rightarrow F\left(x^{\prime} ; z\right)=\frac{a_{x^{\prime}}}{a_{z}} i \Delta_{B}\left(x^{\prime} ; z\right),
\end{gathered}
$$




$$
\mathcal{S}_{C}(x ; z) \Rightarrow F\left(x^{\prime} ; z\right)=i \Delta_{C}\left(x^{\prime} ; z\right) .
$$

Note that each $\mathcal{S}_{F}(x ; z)$ is homogeneous on the first argument $x^{\mu}$. The integral $\mathcal{S}_{A}(x ; z)$ is homogeneous on $z^{\mu}$ as well, and also symmetric under interchange of $x^{\mu}$ and $z^{\mu}$.

\section{B. Absorbing the $B$-type and $C$-type surface terms}

Rather than absorb all the surface terms at once it is simpler to first cancel those of the Other Term, which must also reduce those that remain in the integral term to pure $A$-type (homogeneous on both $x^{\mu}$ and $\left.z^{\mu}\right)$. We accordingly seek a homogeneous gauge parameter $\Delta \theta[A](x)$ which cancels (173). Because this will also change the integral term, we write out the full transformed field,

$$
\hat{A}_{\mu}(x)=\bar{A}_{\mu}(x)-\frac{\partial}{\partial x^{\mu}} \Delta \theta[A](x) .
$$

The propagator of $\hat{A}$ is

$$
\begin{aligned}
\left\langle\Omega\left|T^{*}\left[\hat{A}_{\mu}(x) \hat{A}_{\nu}(z)\right]\right| \Omega\right\rangle= & \left\langle\Omega\left|T^{*}\left[\bar{A}_{\mu}(x) \bar{A}_{\nu}(z)\right]\right| \Omega\right\rangle-\frac{\partial}{\partial x^{\mu}}\left\langle\Omega\left|T^{*}\left[\Delta \theta(x) A_{\nu}(z)\right]\right| \Omega\right\rangle \\
& -\frac{\partial}{\partial z^{\nu}}\left\langle\Omega\left|T^{*}\left[A_{\mu}(x) \Delta \theta(z)\right]\right| \Omega\right\rangle+\frac{\partial}{\partial x^{\mu}} \frac{\partial}{\partial z^{\nu}}\langle\Omega| T^{*}[\Delta \theta(x) \bar{\theta}(z)+\bar{\theta}(x) \Delta \theta(z) \\
& +\Delta \theta(x) \Delta \theta(z)]|\Omega\rangle .
\end{aligned}
$$

The terms on the final line of (180) must belong to the integral term (174), and most of the middle line of (180) must similarly belong to the other term (173). If we assume $\Delta \theta[A](x)$ depends only on $A_{0}$, then the break is clean and we have

$$
\left\langle\Omega\left|T^{*}\left[\Delta \theta(x) A_{0}(z)\right]\right| \Omega\right\rangle=\frac{a_{z} \mathcal{S}_{C}(x ; z)}{(D-3) H},
$$

$$
\begin{aligned}
\langle\Omega| T^{*} & {[\Delta \theta(x) \bar{\theta}(z)+\bar{\theta}(x) \Delta \theta(z)+\Delta \theta(x) \Delta \theta(z)]|\Omega\rangle } \\
& =(A \text {-type terms })-\left[\frac{\mathcal{S}_{C}(x ; z)+\mathcal{S}_{C}(z ; x)}{2(D-3)^{2} H^{2}}\right]-\int_{V} d^{D} x^{\prime} \sqrt{-g\left(x^{\prime}\right)} G\left(x ; x^{\prime}\right)\left[\mathcal{S}_{B}\left(z ; x^{\prime}\right)+\frac{\mathcal{S}_{C}\left(z ; x^{\prime}\right)}{D-3}\right] \\
& -\int_{V} d^{D} z^{\prime} \sqrt{-g\left(z^{\prime}\right)} G\left(z ; z^{\prime}\right)\left[\mathcal{S}_{B}\left(x ; z^{\prime}\right)+\frac{\mathcal{S}_{C}\left(x ; z^{\prime}\right)}{D-3}\right] .
\end{aligned}
$$

It is straightforward to see that relation (181) fixes the homogeneous part of the gauge parameter to be

$$
\Delta \theta(x)=\frac{-1}{(D-3) H} \int_{\partial V} d^{D-1} x_{\rho}^{\prime} \sqrt{-g^{\prime}} g^{\prime \rho \sigma}\left[\frac{A_{0}\left(x^{\prime}\right)}{a_{x^{\prime}}} \partial_{\sigma}^{\prime} G\left(x ; x^{\prime}\right)-G\left(x ; x^{\prime}\right) \partial_{\sigma}^{\prime} \frac{A_{0}\left(x^{\prime}\right)}{a_{x^{\prime}}}\right] .
$$

Combining (168) and (183) gives

$$
\left\langle\Omega\left|T^{*}[\Delta \theta(x) \bar{\theta}(z)]\right| \Omega\right\rangle=\frac{-1}{(D-3) H} \int_{V} d^{D} z^{\prime} G\left(z ; z^{\prime}\right) \frac{\partial}{\partial z^{\prime 0}}\left[a_{z^{\prime}}^{D-1} \mathcal{S}_{C}\left(x ; z^{\prime}\right)\right] .
$$

The surface integral $\mathcal{S}_{C}\left(x ; z^{\prime}\right)$ has the form (175) with the function $F\left(x^{\prime} ; z^{\prime}\right)=i \Delta_{C}\left(x^{\prime} ; z^{\prime}\right)$. Multiplying this by the factor of $a_{z^{\prime}}^{D-1}$ and taking the derivative gives an expression which we can simplify using relations (124) and (90), 


$$
\begin{gathered}
\frac{\partial}{\partial z^{0}}\left[a_{z^{\prime}}^{D-1} i \Delta_{C}\left(x^{\prime} ; z^{\prime}\right)\right]=H a_{z^{\prime}}^{D}\left\{(D-1) C-(2-y) C^{\prime}+2 \frac{a_{x^{\prime}}}{a_{z^{\prime}}} C^{\prime}\right\} \\
=H a_{z^{\prime}}^{D}\left\{2 C+(D-3) C-\frac{1}{2}(D-3)(2-y) B+(D-3) \frac{a_{x^{\prime}}}{a_{z^{\prime}}} B-(2-y) A^{\prime}+2 \frac{a_{x^{\prime}}}{a_{z^{\prime}}} A^{\prime}\right\} \\
=H a_{z^{\prime}}^{D}\left\{2 C+(D-3) \frac{a_{x^{\prime}}}{a_{z^{\prime}}} B\right\}+a_{z^{\prime}}^{D-1} \frac{\partial}{\partial z^{\prime 0}}\left[i \Delta_{A}\left(x^{\prime} ; z^{\prime}\right)\right] .
\end{gathered}
$$

The final term involving $i \Delta_{A}\left(x^{\prime} ; z^{\prime}\right)$ gives rise to an $A$-type surface term whose form we will work out in the next subsection. We can therefore write,

$$
\left\langle\Omega\left|T^{*}[\Delta \theta(x) \bar{\theta}(z)]\right| \Omega\right\rangle=(A \text {-type terms })-\int_{V} d^{D} z^{\prime} \sqrt{-g\left(z^{\prime}\right)} G\left(z ; z^{\prime}\right)\left[\mathcal{S}_{B}\left(x ; z^{\prime}\right)+\frac{2}{D-3} \mathcal{S}_{C}\left(x ; z^{\prime}\right)\right] .
$$

Interchanging $x^{\mu}$ and $z^{\mu}$ gives

$$
\left\langle\Omega\left|T^{*}[\bar{\theta}(x) \Delta \theta(z)]\right| \Omega\right\rangle=(A \text {-type terms })-\int_{V} d^{D} x^{\prime} \sqrt{-g\left(x^{\prime}\right)} G\left(x ; x^{\prime}\right)\left[\mathcal{S}_{B}\left(z ; x^{\prime}\right)+\frac{2}{D-3} \mathcal{S}_{C}\left(z ; x^{\prime}\right)\right] .
$$

The term with two $\Delta \theta$ 's yields a surface integral of surface integrals that we can write as a volume integral of surface integrals using Green's second identity,

$$
\begin{aligned}
\left\langle\Omega\left|T^{*}[\Delta \theta(x) \Delta \theta(z)]\right| \Omega\right\rangle= & -\frac{1}{(D-3)^{2} H^{2}} \int_{\partial V} d^{D-1} x_{\rho}^{\prime} \sqrt{-g\left(x^{\prime}\right)} g^{\rho \sigma}\left(x^{\prime}\right) \times\left[\mathcal{S}_{C}\left(z ; x^{\prime}\right) \frac{\partial}{\partial x^{\prime \sigma}} G\left(x ; x^{\prime}\right)\right. \\
& \left.-G\left(x ; x^{\prime}\right) \frac{\partial}{\partial x^{\prime} \sigma} \mathcal{S}_{C}\left(z ; x^{\prime}\right)\right] \\
= & \frac{-1}{(D-3)^{2} H^{2}} \int_{V} d^{D} x^{\prime} \sqrt{-g\left(x^{\prime}\right)}\left[\mathcal{S}_{C}\left(z ; x^{\prime}\right) \square_{x^{\prime}} G\left(x ; x^{\prime}\right)-G\left(x ; x^{\prime}\right) \square_{x^{\prime}} \mathcal{S}_{C}\left(z ; x^{\prime}\right)\right]
\end{aligned}
$$

Of course, we can use the identity $\sqrt{-g\left(x^{\prime}\right)} \square_{x^{\prime}} G\left(x ; x^{\prime}\right)=i \delta^{D}\left(x-x^{\prime}\right)$, and the quantity $\sqrt{-g\left(x^{\prime}\right)} \square_{x^{\prime}} \mathcal{S}_{C}\left(z ; x^{\prime}\right)$ involves

$$
\sqrt{-g\left(x^{\prime}\right)} \square_{x^{\prime}} i \Delta_{C}\left(z^{\prime} ; x^{\prime}\right)=i \delta^{D}\left(x^{\prime}-z^{\prime}\right)+2(D-3) H^{2} \sqrt{-g\left(x^{\prime}\right)} i \Delta_{C}\left(z^{\prime} ; x^{\prime}\right) .
$$

The delta function in (192) gives another A-type term whose form we work out in the next subsection. Hence, we have

$$
\left\langle\Omega\left|T^{*}[\Delta \theta(x) \Delta \theta(z)]\right| \Omega\right\rangle=(A \text {-type terms })-\frac{\mathcal{S}_{C}(z ; x)}{(D-3)^{2} H^{2}}+\frac{2}{D-3} \int_{V} d^{D} x^{\prime} \sqrt{-g\left(x^{\prime}\right)} G\left(x ; x^{\prime}\right) \mathcal{S}_{C}\left(z ; x^{\prime}\right) .
$$

The result is symmetric in $x^{\mu}$ and $z^{\mu}$ so we can express it as 


$$
\begin{aligned}
\left\langle\Omega\left|T^{*}[\Delta \theta(x) \Delta \theta(z)]\right| \Omega\right\rangle= & (A \text {-type terms })-\frac{\mathcal{S}_{C}(x ; z)+\mathcal{S}_{C}(z ; x)}{2(D-3)^{2} H^{2}}+\int_{V} d^{D} x^{\prime} \sqrt{-g\left(x^{\prime}\right)} G\left(x ; x^{\prime}\right) \frac{\mathcal{S}_{C}\left(z ; x^{\prime}\right)}{D-3} \\
& +\int_{V} d^{D} z^{\prime} \sqrt{-g\left(z^{\prime}\right)} G\left(z ; z^{\prime}\right) \frac{\mathcal{S}_{C}\left(x ; z^{\prime}\right)}{D-3} .
\end{aligned}
$$

Combining expressions (188) and (189) with (194) gives the desired form (182),

$$
\begin{aligned}
\langle\Omega| T^{*} & {[\Delta \theta(x) \bar{\theta}(z)+\bar{\theta}(x) \Delta \theta(z)+\Delta \theta(x) \Delta \theta(z)]|\Omega\rangle } \\
& =(A \text {-type terms })-\left[\frac{\mathcal{S}_{C}(x ; z)+\mathcal{S}_{C}(z ; x)}{2(D-3)^{2} H^{2}}\right]-\int_{V} d^{D} x^{\prime} \sqrt{-g\left(x^{\prime}\right)} G\left(x ; x^{\prime}\right)\left[\mathcal{S}_{B}\left(z ; x^{\prime}\right)+\frac{\mathcal{S}_{C}\left(z ; x^{\prime}\right)}{D-3}\right] \\
& -\int_{V} d^{D} z^{\prime} \sqrt{-g\left(z^{\prime}\right)} G\left(z ; z^{\prime}\right)\left[\mathcal{S}_{B}\left(x ; z^{\prime}\right)+\frac{\mathcal{S}_{C}\left(x ; z^{\prime}\right)}{D-3}\right] .
\end{aligned}
$$

\section{Absorbing the A-type surface terms}

We should begin this section by clarifying precisely what the $A$-type surface terms are. They reside entirely in the integral term, and they consist of $\mathcal{S}_{A} /(D-3) H^{2}$ plus the $A$-type surface terms induced by the gauge parameter $\Delta \theta[A]$. We first reduce $\mathcal{S}_{A}$ to a pair of temporal surface terms, and then derive similar expressions for the $A$-type surface terms from $\Delta \theta[A]$. This will motivate our construction of the final gauge parameter $\delta \theta[A]$, which absorbs the $A$-type surface terms and gives full agreement with the invariant propagator.

Recall that the surface integral $\mathcal{S}_{A}(x ; z)$ is

$$
\mathcal{S}_{A}(x ; z) \equiv \int_{\partial V} d^{D-1} x_{\rho}^{\prime} \sqrt{-g^{\prime}} g^{\prime \rho \sigma}\left[i \Delta_{A^{\prime}}\left(x^{\prime} ; z\right) \partial_{\sigma}^{\prime} G\left(x ; x^{\prime}\right)-G\left(x ; x^{\prime}\right) \partial_{\sigma}^{\prime} i \Delta_{A^{\prime}}\left(x^{\prime} ; z\right)\right]
$$

where $i \Delta_{A^{\prime}}(x ; z)$ is the derivative with respect to $\nu$ [evaluated at $\nu=(D-1) / 2$ ] of the Fourier mode sum,

$$
i \Delta_{\nu}(x ; z)=\int \frac{d^{D-1} k}{(2 \pi)^{D-1}} e^{i \vec{k} \cdot(\vec{x}-\vec{z})}\left\{\theta\left(x^{0}-z^{0}\right) u_{\nu}\left(x^{0}, k\right) u_{\nu}^{*}\left(z^{0}, k\right)+\theta\left(z^{0}-x^{0}\right) u_{\nu}^{*}\left(x^{0}, k\right) u_{\nu}\left(z^{0}, k\right)\right\} .
$$

Because $G(x ; z)$ is $-i$ times the same mode sum [again evaluated at $\nu=(D-1) / 2$ ], we see that the surface terms at spatial infinity make no contribution. One can therefore express $\mathcal{S}_{A}(x ; z)$ as a Fourier mode sum of temporal surface terms

$$
\mathcal{S}_{A}(x ; z)=i \int \frac{d^{D-1} k}{(2 \pi)^{D-1}} e^{i \vec{k} \cdot(\vec{x}-\vec{z})} \times\left\{u_{A}^{*}\left(x^{0}, k\right) u_{A}^{*}\left(z^{0}, k\right) \times \mathcal{F}\left(-k \eta_{2}\right)-u_{A}\left(x^{0}, k\right) u_{A}\left(z^{0}, k\right) \times \mathcal{F}^{*}\left(-k \eta_{1}\right)\right\},
$$

where $\eta_{1}$ and $\eta_{2}$ are the initial and final times, respectively, and the function $\mathcal{F}(-k \eta)$ is

$$
\mathcal{F}(-k \eta) \equiv a^{D-2}\left\{\frac{\partial u_{\nu}(\eta, k)}{\partial \nu} \frac{\partial u_{\nu}(\eta, k)}{\partial \eta}-u_{\nu}(\eta, k) \frac{\partial^{2} u_{\nu}(\eta, k)}{\partial \nu \partial \eta}\right\}_{\nu=(D-1) / 2}
$$




$$
=\frac{\pi}{4 H a}\left\{\frac{\partial H_{\nu}^{(1)}(-k \eta)}{\partial \nu} \frac{\partial H_{\nu}^{(1)}(-k \eta)}{\partial \eta}-H_{\nu}^{(1)}(-k \eta) \frac{\partial^{2} H_{\nu}^{(1)}(-k \eta)}{\partial \nu \partial \eta}\right\}_{\nu=(D-1) / 2} .
$$

This function $\mathcal{F}(z)$ has the interesting property that it can be related to the product of two Hankel functions, without any derivatives with respect to the index or the argument. ${ }^{33}$ To see the relation we define,

$$
\begin{gathered}
\mathcal{E}_{\nu}(z) \equiv z\left[H_{\nu}^{(1)}(z)\right]^{2} \\
\mathcal{G}_{\nu}(z) \equiv z\left[\partial_{\nu} H_{\nu}^{(1)}(z) \partial_{z} H_{\nu}^{(1)}(z)-H_{\nu}^{(1)}(z) \partial_{\nu} \partial_{z} H_{\nu}^{(1)}(z)\right] .
\end{gathered}
$$

Of course, we have

$$
\mathcal{F}(-k \eta)=-\frac{\pi}{4} \times \mathcal{G}_{\nu}(-k \eta)
$$

and the relation to $\mathcal{E}_{\nu}$ is ${ }^{33}$

$$
\partial_{z} \mathcal{G}_{\nu}(z)=-\frac{2 \nu}{z^{2}} \mathcal{E}_{\nu}(z)
$$

The integration constant can be fixed using the asymptotic expansion for large $z$ to give

$$
\mathcal{G}_{\nu}(z)=2 \nu \int_{z}^{\infty} d z^{\prime} \frac{\mathcal{E}_{\nu}\left(z^{\prime}\right)}{z^{\prime 2}}
$$

The key identity for $\Delta \theta[A]$ to produce $A$-type surface terms is

$$
\begin{aligned}
\frac{\partial}{\partial x^{\prime 0}} \frac{\partial}{\partial z^{\prime 0}} i \Delta_{C}\left(x^{\prime} ; z^{\prime}\right)= & \frac{i}{a_{x^{\prime}}^{D-2}} \delta^{D}\left(x^{\prime}-z^{\prime}\right)+\frac{\partial^{2} y\left(x^{\prime} ; z^{\prime}\right)}{\partial x^{\prime 0} \partial z^{\prime 0}} C^{\prime}\left(y\left(x^{\prime} ; z^{\prime}\right)\right) \\
& +\frac{\partial y\left(x^{\prime} ; z^{\prime}\right)}{\partial x^{\prime 0}} \frac{\partial y\left(x^{\prime} ; z^{\prime}\right)}{\partial z^{\prime 0}} C^{\prime \prime}\left(y\left(x^{\prime} ; z^{\prime}\right)\right) .
\end{aligned}
$$

The $A$-type surface terms come exclusively from the delta function term; the other contributions produce $B$-type and $C$-type surface terms we have already included. Note that because one gets a $D$-dimensional delta function, whereas the initial and final surface integrals are only $(D-1)$-dimensional, it is necessary to regulate $\Delta \theta[A]$ to make the $A$-type surface term well defined. An obvious regularization is to integrate the initial and final time surfaces over a small range of duration $\Delta \eta=2 \epsilon$,

$$
\begin{aligned}
\Delta \theta_{\epsilon}(x) \equiv & \frac{1}{2 \epsilon(D-3) H}\left[\int_{\eta_{2}-\epsilon}^{\eta_{2}+\epsilon} d x^{\prime 0}-\int_{\eta_{1}-\epsilon}^{\eta_{1}+\epsilon} d x^{\prime 0}\right] \int d^{D-1} \vec{x}^{\prime} \times a_{x^{\prime}}^{D-2}\left\{\frac{1}{a_{x^{\prime}}} A_{0}\left(x^{\prime}\right) \frac{\partial}{\partial x^{\prime 0}} G\left(x ; x^{\prime}\right)\right. \\
& \left.-G\left(x ; x^{\prime}\right) \frac{\partial}{\partial x^{\prime 0}}\left[\frac{1}{a_{x^{\prime}}} A_{0}\left(x^{\prime}\right)\right]\right\} .
\end{aligned}
$$

Let us now work out the $A$-type surface term from $\Delta \theta_{\epsilon}(x) \times \bar{\theta}(z)$. The full expectation value is 


$$
\begin{aligned}
\left\langle\Omega\left|T^{*}\left[\Delta \theta_{\epsilon}(x) \bar{\theta}(z)\right]\right| \Omega\right\rangle= & {\left[\int_{\eta_{2}-\epsilon}^{\eta_{2}+\epsilon} d x^{\prime 0}-\int_{\eta_{1}-\epsilon}^{\eta_{1}+\epsilon} d x^{\prime 0}\right] \int d^{D-1} \vec{x}^{\prime} \int_{V} d^{D} z^{\prime} \frac{a_{x^{\prime}}^{D-2} G\left(z ; z^{\prime}\right)}{2 \epsilon(D-3) H} } \\
& \times \frac{\partial}{\partial z^{\prime 0}}\left\{a_{z^{\prime}}^{D-1} i \Delta_{C}\left(x^{\prime} ; z^{\prime}\right) \frac{\partial}{\partial x^{\prime 0}} G\left(x ; x^{\prime}\right)-a_{z^{\prime}}^{D-1} G\left(x ; x^{\prime}\right) \frac{\partial}{\partial x^{\prime 0}} i \Delta_{C}\left(x^{\prime} ; z^{\prime}\right)\right\} .
\end{aligned}
$$

However, we already accounted for most of this in the previous subsection; it is only the delta function from using (206) on the final surface term which makes the new contribution we seek,

$$
\begin{aligned}
\left\langle\Omega\left|T^{*}\left[\Delta \theta_{\epsilon}(x) \bar{\theta}(z)\right]\right| \Omega\right\rangle_{A-\text { type }}= & \frac{-i}{2 \epsilon(D-3) H}\left[\int_{\eta_{2}-\epsilon}^{\eta_{2}+\epsilon} d x^{\prime 0}-\int_{\eta_{1}-\epsilon}^{\eta_{1}+\epsilon} d x^{\prime 0}\right] \\
& \times \int d^{D-1} \vec{x}^{\prime} \int_{V} d^{D} z^{\prime} G\left(z ; z^{\prime}\right) a_{z^{\prime}}^{D-1} G\left(x ; x^{\prime}\right) \delta^{D}\left(x^{\prime}-z^{\prime}\right) .
\end{aligned}
$$

The integration over $z^{\prime \mu}$ is not affected by our regularization of $\Delta \theta_{\epsilon}(x)$,

$$
\int d^{D} z^{\prime} \equiv \int_{\eta_{1}}^{\eta_{2}} d z^{\prime 0} \int d^{D-1} \vec{z}^{\prime}
$$

It is therefore only half of the $x^{\prime 0}$ range over which the delta function can be saturated. Taking the unregulated limit gives

$$
\begin{gathered}
\lim _{\epsilon \rightarrow 0}\left\langle\Omega\left|T^{*}\left[\Delta \theta_{\epsilon}(x) \bar{\theta}(z)\right]\right| \Omega\right\rangle_{A-\mathrm{type}}=\lim _{\epsilon \rightarrow 0} \frac{-i}{2 \epsilon(D-3) H}\left[\int_{\eta_{2}-\epsilon}^{\eta_{2}} d x^{\prime 0}\right. \\
\left.-\int_{\eta_{1}}^{\eta_{1}+\epsilon} d x^{\prime 0}\right] \int d^{D-1} \vec{x}^{\prime} G\left(z ; x^{\prime}\right) a_{x^{\prime}}^{D-1} G\left(x ; x^{\prime}\right) \\
=\frac{i}{2(D-3) H} \int d^{D-1} \vec{x}^{\prime}\left[a_{x^{\prime}}^{D-1} i \Delta_{A}\left(x ; x^{\prime}\right) i \Delta_{A}\left(z ; x^{\prime}\right)\right]_{x^{\prime}=\eta_{1}}^{x^{\prime 0}=\eta_{2}} .
\end{gathered}
$$

One obviously gets the same result (212) from $\bar{\theta}(x) \times \Delta \theta_{\epsilon}(z)$ so the total for these "mixed" terms is

$$
\begin{aligned}
& \lim _{\epsilon \rightarrow 0}\left\langle\Omega\left|T^{*}\left[\Delta \theta_{\epsilon}(x) \bar{\theta}(z)+\bar{\theta}(x) \Delta \theta_{\epsilon}(z)\right]\right| \Omega\right\rangle_{A-\text { type }}=\frac{i}{(D-3) H} \int d^{D-1} \vec{x}^{\prime}\left[a_{x^{\prime}}^{D-1} i \Delta_{A}\left(x ; x^{\prime}\right) i \Delta_{A}\left(z ; x^{\prime}\right)\right]_{x^{\prime}=\eta_{1}}^{x^{\prime 0}=\eta_{2}} \\
= & \frac{i}{(D-3) H} \int \frac{d^{D-1} k}{(2 \pi)^{D-1}} e^{i \vec{k} \cdot(\vec{x}-\vec{z})}\left\{a_{2}^{D-1} u_{A}^{*}\left(x^{0}, k\right) u_{A}^{*}\left(z^{0}, k\right)\left[u_{A}\left(\eta_{2}, k\right)\right]^{2}-a_{1}^{D-1} u_{A}\left(x^{0}, k\right) u_{A}\left(z^{0}, k\right)\left[u_{A}^{*}\left(\eta_{1}, k\right)\right]^{2}\right\} \\
= & \frac{i}{(D-3) H^{2}} \int \frac{d^{D-1} k}{(2 \pi)^{D-1}} e^{i \vec{k} \cdot(\vec{x}-\vec{z})}\left\{u_{A}^{*}\left(x^{0}, k\right) u_{A}^{*}\left(z^{0}, k\right) \times \frac{\pi}{4}\left[H_{\nu}^{(1)}\left(-k \eta_{2}\right)\right]^{2}=-u_{A}\left(x^{0}, k\right) u_{A}\left(z^{0}, k\right) \times \frac{\pi}{4}\left[H_{\nu}^{(1)}\right.\right. \\
& \left.\left.\times\left(-k \eta_{2}\right)\right]^{* 2}\right\} .
\end{aligned}
$$

Expression (215) combines nicely with the $A$-type surface term from $\bar{\theta}$ 


$$
\begin{aligned}
\frac{\mathcal{S}_{A}(x ; z)}{(D-3) H^{2}}= & \frac{i}{(D-3) H^{2}} \int \frac{d^{D-1} k}{(2 \pi)^{D-1}} e^{i \vec{k} \cdot(\vec{x}-\vec{z})} \times\left\{u_{A}^{*}\left(x^{0}, k\right) u_{A}^{*}\left(z^{0}, k\right) \times \mathcal{F}\left(-k \eta_{2}\right)-u_{A}\left(x^{0}, k\right) u_{A}\left(z^{0}, k\right)\right. \\
& \left.\times \mathcal{F}^{*}\left(-k \eta_{1}\right)\right\}
\end{aligned}
$$

By partial integration, we can express $\mathcal{F}(z)$ as

$$
\begin{gathered}
\mathcal{F}(z)=-\frac{\pi}{4} \times 2 \nu \int_{z}^{\infty} d z^{\prime} \frac{1}{z^{\prime}}\left[H_{\nu}^{(1)}\left(z^{\prime}\right)\right]^{2} \\
=-\frac{\pi}{4} \times\left[H_{\nu}^{(1)}(z)\right]^{2}-\frac{\pi}{4} \times \int_{z}^{\infty} d z^{\prime} \frac{1}{z^{\prime 2 \nu}} \frac{\partial}{\partial z^{\prime}}\left[z^{\prime \nu} H_{\nu}^{(1)}\left(z^{\prime}\right)\right]^{2} .
\end{gathered}
$$

So the first term of (218) is canceled by (215).

The full expectation value for $\Delta \theta_{\epsilon}(x) \times \Delta \theta_{\epsilon}(z)$ is

$$
\begin{aligned}
\left\langle\Omega\left|T^{*}\left[\Delta \theta_{\epsilon}(x) \Delta \theta_{\epsilon}(z)\right]\right| \Omega\right\rangle= & \frac{-1}{4 \epsilon^{2}(D-3)^{2} H^{2}} \times\left[\int_{\eta_{2}-\epsilon}^{\eta_{2}+\epsilon} d x^{\prime 0}-\int_{\eta_{1}-\epsilon}^{\eta_{1}+\epsilon} d x^{\prime 0}\right] \int d^{D-1} \vec{x}^{\prime} a_{x^{\prime}}^{D-2}\left[\int_{\eta_{2}-\epsilon}^{\eta_{2}+\epsilon} d z^{\prime 0}\right. \\
& \left.-\int_{\eta_{1}-\epsilon}^{\eta_{1}+\epsilon} d z^{\prime 0}\right] \int d^{D-1} \vec{z}^{\prime} a_{z^{\prime}}^{D-2} \times\left\{i \Delta_{C}\left(x^{\prime} ; z^{\prime}\right) \frac{\partial G\left(x ; x^{\prime}\right)}{\partial x^{\prime 0}} \frac{\partial G\left(z ; z^{\prime}\right)}{\partial z^{\prime 0}}\right. \\
& -G\left(z ; z^{\prime}\right) \frac{\partial G\left(x ; x^{\prime}\right)}{\partial x^{\prime 0}} \frac{\partial i \Delta_{C}\left(x^{\prime} ; z^{\prime}\right)}{\partial z^{\prime 0}}-G\left(x ; x^{\prime}\right) \frac{\partial G\left(z ; z^{\prime}\right)}{\partial z^{\prime 0}} \frac{\partial i \Delta_{C}\left(x^{\prime} ; z^{\prime}\right)}{\partial x^{\prime 0}} \\
& \left.+G\left(x ; x^{\prime}\right) G\left(z ; z^{\prime}\right) \frac{\partial^{2} i \Delta_{C}\left(x^{\prime} ; z^{\prime}\right)}{\partial x^{\prime 0} \partial z^{\prime 0}}\right\} .
\end{aligned}
$$

As with the mixed term (212), we have already reduced most of this in the previous subsection. The only new contribution is derived from the delta function one obtains by using (206) on the final term

$$
\begin{aligned}
\left\langle\Omega\left|T^{*}\left[\Delta \theta_{\epsilon}(x) \Delta \theta_{\epsilon}(z)\right]\right| \Omega\right\rangle_{A-\text { type }}= & \frac{-i}{4 \epsilon^{2}(D-3)^{2} H^{2}}\left[\int_{\eta_{2}-\epsilon}^{\eta_{2}+\epsilon} d x^{\prime 0}-\int_{\eta_{1}-\epsilon}^{\eta_{1}+\epsilon} d x^{\prime 0}\right] \int d^{D-1} \vec{x}^{\prime} a_{x^{\prime}}^{D-2} \\
& \times\left[\int_{\eta_{2}-\epsilon}^{\eta_{2}+\epsilon} d z^{\prime 0}-\int_{\eta_{1}-\epsilon}^{\eta_{1}+\epsilon} d z^{\prime 0}\right] \int d^{D-1} \vec{z}^{\prime} G\left(x ; x^{\prime}\right) G\left(z ; z^{\prime}\right) \delta^{D}\left(x^{\prime}-z^{\prime}\right) \\
= & \frac{i}{4 \epsilon^{2}(D-3)^{2} H^{2}}\left[\int_{\eta_{2}-\epsilon}^{\eta_{2}+\epsilon} d x^{\prime 0}-\int_{\eta_{1}-\epsilon}^{\eta_{1}+\epsilon} d x^{\prime 0}\right] \int d^{D-1} \vec{x}^{\prime} a_{x^{\prime}}^{D-2} i \Delta_{A}\left(x ; x^{\prime}\right) i \Delta_{A}\left(z ; x^{\prime}\right) \\
= & \frac{i}{4 \epsilon^{2}(D-3)^{2} H^{2}} \int \frac{d^{D-1} k}{(2 \pi)^{D-1}} e^{i \vec{k} \cdot(\vec{x}-\vec{z})}\left\{u_{A}^{*}\left(x^{0}, k\right) u_{A}^{*}\left(z^{0}, k\right) \int_{\eta_{2}-\epsilon}^{\eta_{2}+\epsilon} d \eta a_{\eta}^{D-2}\left[u_{A}(\eta, k)\right]^{2}\right. \\
& \left.-u_{A}\left(x^{0}, k\right) u_{A}\left(z^{0}, k\right) \int_{\eta_{1}-\epsilon}^{\eta_{1}+\epsilon} d \eta a_{\eta}^{D-2}\left[u_{A}^{*}(\eta, k)\right]^{2}\right\}
\end{aligned}
$$

The integrations with respect to $\eta$ can be performed, but it is not possible to take the unregulated limit, 


$$
\begin{gathered}
\int_{\eta_{2}-\epsilon}^{\eta_{2}+\epsilon} d \eta a_{\eta}^{D-2}\left[u_{A}(\eta, k)\right]^{2}=-\frac{\pi}{4} \int_{\eta_{2}-\epsilon}^{\eta_{2}+\epsilon} d \eta \eta\left[H_{\nu}^{(1)}(-k \eta)\right]^{2} \\
=-\frac{\pi}{4 k^{2}} \int_{-k\left(\eta_{2}-\epsilon\right)}^{-k\left(\eta_{2}+\epsilon\right)} d z z\left[H_{\nu}^{(1)}(z)\right]^{2} \\
=-\left.\frac{\pi}{8 k^{2}}\left\{\left(z^{2}-\nu^{2}\right)\left[H_{\nu}^{(1)}(z)\right]^{2}+z^{2}\left[\frac{\partial}{\partial z} H_{\nu}^{(1)}(z)\right]^{2}\right\}\right|_{z=-k\left(\eta_{2}-\epsilon\right)} ^{z=-k\left(\eta_{2}+\epsilon\right)} \\
=-\frac{\pi}{4} \times 2 \epsilon \times \eta_{2}\left[H_{\nu}^{(1)}\left(-k \eta_{2}\right)\right]^{2}+O\left(\epsilon^{3}\right) .
\end{gathered}
$$

Combining the various $A$-type surface terms gives a result of the form,

$$
\begin{aligned}
(A \text {-type terms })= & \frac{-i}{(D-3) H^{2}} \int \frac{d^{D-1} k}{(2 \pi)^{D-1}} e^{i \vec{k} \cdot(\vec{x}-\vec{z})} \times\left\{u_{A}^{*}\left(x^{0}, k\right) u_{A}^{*}\left(z^{0}, k\right) \mathcal{A}\left(\eta_{2}, k, \epsilon\right)\right. \\
& \left.-u_{A}\left(x^{0}, k\right) u_{A}\left(z^{0}, k\right) \mathcal{A}^{*}\left(\eta_{1}, k, \epsilon\right)\right\} .
\end{aligned}
$$

The function $\mathcal{A}(\eta, k, \epsilon)$ is

$$
\mathcal{A}(\eta, k, \epsilon)=\frac{\pi}{4}\left\{\frac{\eta\left[H_{\nu}^{(1)}(-k \eta)\right]^{2}}{2 \epsilon(D-3)}+\int_{-k \eta}^{\infty} d z^{\prime} \frac{1}{z^{\prime 2 \nu}} \frac{\partial}{\partial z^{\prime}}\left[z^{\prime \nu} H_{\nu}^{(1)}\left(z^{\prime}\right)\right]^{2}+O(\epsilon)\right\} .
$$

We seek a homogeneous gauge parameter $\delta \theta[A](x)$ that cancels (227) and (228), in the limit that $\epsilon$ goes to zero, without changing the Other Term. If we construct it from $A_{i}$ rather than $A_{0}$, then there will be no interference between $\Delta \theta[A]$ and $\delta \theta[A]$. Suppose further that $\delta \theta[A](x)$, like $\Delta \theta[A](x)$ involves an integral over a dummy variable $x^{\prime \mu}$, and that the field $A_{i}\left(x^{\prime}\right)$ is differentiated with respect to $x^{\prime 0}$. What we want is that the expectation value of $\delta \theta[A](x) \times A_{i}(z)$ is zero unless there is also a derivative with respect to $z^{0}$. If we can construct a $\delta \theta[A]$ with this property then the only nonzero contribution to the transformed propagator will come from $\delta \theta[A](x) \times \delta \theta[A](z)$.

It is simplest to construct the term we want by analogy with the simple harmonic oscillator, whose Heisenberg position operator is

$$
q(t)=\frac{1}{\sqrt{2 m \omega}}\left[a e^{-i \omega t}+a^{\dagger} e^{i \omega t}\right] .
$$

Note that we can isolate the raising and lower operators by taking linear combinations of $\dot{q}$ and $i \omega q$,

$$
\dot{q}(t)+i \omega q(t)=\frac{2 i \omega}{\sqrt{2 m \omega}} a^{\dagger} e^{i \omega t}, \quad \dot{q}(t)-i \omega q(t)=\frac{-2 i \omega}{\sqrt{2 m \omega}} a e^{-i \omega t} .
$$

We assume the usual commutation relations and ground state $|\Omega\rangle$,

$$
\left[a, a^{\dagger}\right]=1, \quad a|\Omega\rangle=0=\langle\Omega| a^{\dagger} .
$$

If $t$ comes before the last time $t_{2}$, and after the earliest time $t_{1}$, then we have

$$
\left\langle\Omega\left|T^{*}\left[\left(\dot{q}\left(t_{2}\right)+i \omega q\left(t_{2}\right)\right) q(t)\right]\right| \Omega\right\rangle=0 \quad \forall \quad t<t_{2},
$$




$$
\left\langle\Omega\left|T^{*}\left[\left(\dot{q}\left(t_{1}\right)-i \omega q\left(t_{1}\right)\right) q(t)\right]\right| \Omega\right\rangle=0 \quad \forall \quad t_{1}<t .
$$

The only nonzero expectation value comes from the $T^{*}$-ordered product of two factors of the combination (230),

$$
\begin{aligned}
\left\langle\Omega\left|T^{*}\left[\left(\dot{q}\left(t_{2}\right)+i \omega q\left(t_{2}\right)\right)\left(\dot{q}\left(t_{2}^{\prime}\right)+i \omega q\left(t_{2}^{\prime}\right)\right)\right]\right| \Omega\right\rangle & =\frac{i}{m} \delta\left(t_{2}-t_{2}^{\prime}\right), \\
\left\langle\Omega\left|T^{*}\left[\left(\dot{q}\left(t_{1}\right)-i \omega q\left(t_{1}\right)\right)\left(\dot{q}\left(t_{1}^{\prime}\right)-i \omega q\left(t_{1}^{\prime}\right)\right)\right]\right| \Omega\right\rangle & =-\frac{i}{m} \delta\left(t_{1}-t_{1}^{\prime}\right) .
\end{aligned}
$$

We construct a gauge parameter $\delta \theta[A](x)$ with the desired properties by analogy. The free field mode sum for $A_{i}(x)$ is ${ }^{10,18}$

$$
A_{i}(x)=\int \frac{d^{D-1} k}{(2 \pi)^{D-1}}\left\{a_{x} u_{B}\left(x^{0}, k\right) e^{i \vec{k} \cdot \vec{x}} \beta_{i}(\vec{k})+a_{x} u_{B}^{*}\left(x^{0}, k\right) e^{-i \vec{k} \cdot \vec{x}} \beta_{i}^{\dagger}(\vec{k})\right\},
$$

where the $u_{B}(\eta, k)$ mode functions are given by (118) with index $\nu=(D-3) / 2$ and the $\beta_{i}(\vec{k})$ are canonically normalized annihilation operators. One can isolate $\beta_{i}(\vec{k})$ by taking the spatial Fourier transform,

$$
\tilde{A}_{i}\left(x^{0}, \vec{k}\right) \equiv \int d^{D-1} \vec{x} e^{-i \vec{k} \cdot \vec{x}} A_{i}\left(x^{0}, \vec{x}\right)
$$

Now form linear combinations analogous to (230),

$$
\begin{gathered}
\frac{1}{a} \widetilde{A}_{i}(\eta, \vec{k}) \frac{\partial}{\partial \eta} u_{B}^{*}(\eta, k)-u_{B}^{*}(\eta, k) \frac{\partial}{\partial \eta}\left[\frac{1}{a} \widetilde{A}_{i}(\eta, \vec{k})\right]=\frac{i \beta_{i}(\vec{k})}{a^{D-2}}, \\
\frac{1}{a} \widetilde{A}_{i}(\eta,-\vec{k}) \frac{\partial}{\partial \eta} u_{B}(\eta, k)-u_{B}(\eta, k) \frac{\partial}{\partial \eta}\left[\frac{1}{a} \widetilde{A}_{i}(\eta,-\vec{k})\right]=-\frac{i \beta_{i}^{\dagger}(\vec{k})}{a^{D-2}} .
\end{gathered}
$$

It follows that the desired gauge parameter is

$$
\begin{aligned}
\delta \theta_{\epsilon}(x)= & \frac{i}{\sqrt{2 \epsilon}(D-3) H} \int \frac{d^{D-1} k}{(2 \pi)^{D-1}} e^{i \vec{k} \cdot \vec{x}}\left\{u_{A}^{*}\left(x^{0}, k\right) \int_{\eta_{2}-\epsilon}^{\eta_{2}+\epsilon} d x^{\prime 0} a_{x^{\prime}}^{D-2}\left[(D-3) \mathcal{A}\left(x^{\prime 0}, k, \epsilon\right)\right]^{1 / 2}\right. \\
& \times\left[\frac{k_{i} \widetilde{A}_{i}\left(x^{\prime 0}, \vec{k}\right)}{k a_{x^{\prime}}} \frac{\partial u_{B}\left(x^{\prime 0}, k\right)}{\partial x^{\prime 0}}-u_{B}\left(x^{\prime 0}, k\right) \frac{\partial}{\partial x^{\prime 0}}\left[\frac{k_{i} \widetilde{A}_{i}\left(x^{\prime 0}, \vec{k}\right)}{k a_{x^{\prime}}}\right]\right]-u_{A}\left(x^{0}, k\right) \int_{\eta_{1}-\epsilon}^{\eta_{1}+\epsilon} d x^{\prime 0} a_{x^{\prime}}^{D-2}[(D \\
& \left.\left.-3) \mathcal{A}^{*}\left(x^{\prime 0}, k, \epsilon\right)\right]^{1 / 2} \times\left[\frac{k_{i} \widetilde{A}_{i}\left(x^{\prime 0}, \vec{k}\right)}{k a_{x^{\prime}}} \frac{\partial u_{B}^{*}\left(x^{\prime 0}, k\right)}{\partial x^{\prime 0}}-u_{B}^{*}\left(x^{\prime 0}, k\right) \frac{\partial}{\partial x^{\prime 0}}\left[\frac{k_{i} \widetilde{A}_{i}\left(x^{\prime 0}, \vec{k}\right)}{k a_{x^{\prime}}}\right]\right]\right\} . \quad(240)
\end{aligned}
$$

\section{DISCUSSION}

There are two generic ways to freeze local symmetries:

- exact gauge fixing, in which the fields are made to obey some equation, and

- average gauge fixing, in which a term is added to the Lagrangian.

We have shown that certain average gauges cannot be derived from the canonical formalism on manifolds such as de Sitter for which there are linearization instabilities. Ignoring this problem in electrodynamics causes the vector potential to possess an unphysical and incorrect part which 
drops out of the field strength but affects interaction energies. This may be the origin of the on-shell singularities found in Feynman gauge for the one loop self-mass-squared of charged scalars on de Sitter. ${ }^{18}$

We have also constructed the field-dependent gauge transformation that enforces exact, Lorentz gauge on de Sitter electrodynamics. This was applied to the photon propagator from a non-de Sitter invariant, average gauge, and the result agrees exactly with the de Sitter invariant solution previously obtained from solving the Lorentz gauge propagator equation..$^{19}$ It was already known from adding the compensating gauge transformation to the naive de Sitter transformation that the propagator in the noninvariant gauge shows no physical breaking of de Sitter invariance. ${ }^{10}$ So the fact that our transformation technique produces an invariant result demonstrates that the technique indeed eliminates unphysical breaking of de Sitter invariance.

In a subsequent work, we will employ the same technique to transform the graviton propagator from a non-de Sitter invariant, average gauge ${ }^{9,10}$ to the exact and de Sitter invariant, de Donder gauge. Adding the compensating transformation shows that the breaking of de Sitter invariance in this propagator is physical, ${ }^{16}$ so the expectation is that the transformation technique will not remove it. Because the transformed propagator will obey a de Sitter invariant gauge condition, this should settle the issue about whether or not free gravitons have any de Sitter invariant states. Note that simply obeying a de Sitter invariant propagator equation does not guarantee a de Sitter invariant solution, as the case of the massless, minimally coupled scalar proves. ${ }^{6}$ Note also that physical graviton modes obey precisely the same equation as the massless, minimally coupled scalar. $^{4}$

Constructing the de Donder gauge propagator is a worthy goal in its own right for two reasons. First, exploiting the gauge condition makes a vast simplification in tensor algebra. ${ }^{15}$ Second, using a de Sitter invariant gauge would preclude the need for noninvariant counterterms even though the actual propagator is not de Sitter invariant. ${ }^{14,15}$

A significant technical result of this paper is the "convolution identity" (131) for integrating the propagator $i \Delta_{A}$ of a massless, minimally coupled scalar up against the propagator $i \Delta_{\nu}$ of a massless scalar with conformal coupling,

$$
\xi=\frac{1}{D(D-1)}\left[\left(\frac{D-1}{2}\right)^{2}-\nu^{2}\right] .
$$

The result follows from Green's second identity,

$$
\begin{aligned}
-i \int_{V} d^{D} x^{\prime} \sqrt{-g\left(x^{\prime}\right)} i \Delta_{A}\left(x ; x^{\prime}\right) i \Delta_{\nu}\left(x^{\prime} ; z\right) & \\
= & {\left[\begin{array}{l}
i \Delta_{\nu}(x ; z)-i \Delta_{A}(x ; z) \\
{\left[\left(\frac{D-1}{2}\right)^{2}-\nu^{2}\right] H^{2}}
\end{array}-i \int_{\partial V} d^{D-1} x_{\rho}^{\prime} \sqrt{-g^{\prime}} g^{\prime \rho \sigma}[\right.} \\
& \left.\times \frac{i \Delta_{\nu}\left(x^{\prime} ; z\right) \partial_{\sigma}^{\prime} i \Delta_{A}\left(x ; x^{\prime}\right)-i \Delta_{A}\left(x ; x^{\prime}\right) \partial_{\sigma}^{\prime} i \Delta_{\nu}\left(x^{\prime} ; z\right)}{\left[\left(\frac{D-1}{2}\right)^{2}-\nu^{2}\right] H^{2}}\right] .
\end{aligned}
$$

We expect this to be of great utility in the subsequent graviton project because field-dependent gauge transformations result in precisely such convolutions.

\section{ACKNOWLEDGMENTS}

This work was partially supported by FQXi Mini Grant No. MGB-08-008, by FOM Grant No. 07PR2522, by Utrecht University, by European Union Grant No. MRTN-CT-2004-512194, by Hellenic grant INTERREG IIIA, by NSF Grant Nos. PHY-0653085 and PHY-0855021, and by the Institute for Fundamental Theory at the University of Florida. 
${ }^{1}$ N. C. Tsamis and R. P. Woodard, Class. Quantum Grav. 18, 83 (2001).

${ }^{2}$ N. C. Tsamis and R. P. Woodard, Class. Quantum Grav. 2, 841 (1985).

${ }^{3}$ S. R. Coleman, Subnucl. Ser. 11, 139 (1975).

${ }^{4}$ L. P. Grishchuk, Sov. Phys. JETP 40, 409 (1975).

${ }^{5}$ A. A. Starobinsky, JETP Lett. 30, 682 (1979); Sov. Astron. Lett. 11, 133 (1985).

${ }^{6}$ B. Allen and A. Folacci, Phys. Rev. D 35, 3771 (1987).

${ }^{7}$ B. Allen and M. Turyn, Nucl. Phys. B 292, 813 (1987); S. W. Hawking, T. Hertog, and N. Turok, Phys. Rev. D 62, 063502 (2000); A. Higuchi and S. S. Kouris, Class. Quantum Grav. 18, 4317 (2001); A. Higuchi and R. H. Weeks, ibid. 20, 3005 (2003).

${ }^{8}$ I. Antoniadis and E. Mottola, J. Math. Phys. 32, 1037 (1991).

${ }^{9}$ N. C. Tsamis and R. P. Woodard, Commun. Math. Phys. 162, 217 (1994).

${ }^{10}$ R. P. Woodard, in Deserfest: A Celebration of the Life and Works of Stanley Deser, edited by J. T. Liu, M. J. Duff, K. S. Stelle, and R. P. Woodard (World Scientific, Hackensack, 2006), p. 339.

${ }^{11}$ N. C. Tsamis and R. P. Woodard, Phys. Lett. B 292, 269 (1992).

${ }^{12}$ N. C. Tsamis and R. P. Woodard, Phys. Rev. D 54, 2621 (1996).

${ }^{13}$ N. C. Tsamis and R. P. Woodard, Ann. Phys. 321, 875 (2006).

${ }^{14}$ S. P. Miao and R. P. Woodard, Class. Quantum Grav. 23, 1721 (2006).

${ }^{15}$ E. O. Kahya and R. P. Woodard, Phys. Rev. D 76, 124005 (2007).

${ }^{16}$ G. Kleppe, Phys. Lett. B 317, 305 (1993).

${ }^{17}$ A. Higuchi and Y. C. Lee, Phys. Rev. D 78, 084031 (2008); M. Faizal and A. Higuchi, ibid. 78, 067502 (2008).

${ }^{18}$ E. O. Kahya and R. P. Woodard, Phys. Rev. D 72, 104001 (2005); 74, 084012 (2006).

${ }^{19}$ N. C. Tsamis and R. P. Woodard, J. Math. Phys. 48, 052306 (2007).

${ }^{20}$ R. P. Woodard, Class. Quantum Grav. 10, 483 (1993).

${ }^{21}$ B. Allen and T. Jacobson, Commun. Math. Phys. 103, 669 (1986).

${ }^{22}$ T. Prokopec, N. C. Tsamis, and R. P. Woodard, Class. Quantum Grav. 24, 201 (2007).

${ }^{23}$ T. Prokopec, N. C. Tsamis, and R. P. Woodard, Ann. Phys. 323, 1324 (2008); Phys. Rev. D 78, 043523 (2008).

${ }^{24}$ T. M. Janssen, S. P. Miao, T. Prokopec, and R. P. Woodard, Class. Quantum Grav. 25, 245013 (2008).

${ }^{25}$ N. A. Chernikov and E. A. Tagirov, Ann. I.H.P. Phys. Theor. A9, 109 (1968).

${ }^{26}$ A. Vilenkin, Nucl. Phys. B 226, 527 (1983).

${ }^{27}$ N. C. Tsamis and R. P. Woodard, Class. Quantum Grav. 11, 2969 (1994).

${ }^{28}$ V. K. Onemli and R. P. Woodard, Class. Quantum Grav. 19, 4607 (2002); Phys. Rev. D 70, 107301 (2004).

${ }^{29}$ T. Brunier, V. K. Onemli, and R. P. Woodard, Class. Quantum Grav. 22, 59 (2005).

${ }^{30}$ E. O. Kahya and V. K. Onemli, Phys. Rev. D 76, 043512 (2007).

${ }^{31}$ S. P. Miao and R. P. Woodard, Phys. Rev. D 74, 044019 (2006).

${ }^{32}$ A. Vilenkin and L. H. Ford, Phys. Rev. D 26, 1231 (1982); A. D. Linde, Phys. Lett. B 116, 335 (1982); A. A. Starobinsky, ibid. 117, 175 (1982).

${ }^{33}$ N. C. Tsamis and R. P. Woodard, Class. Quantum Grav. 20, 5205 (2003). 